\title{
Spectral (Isotropic) Manifolds and Their Dimension
}

\author{
Aris DANIILIDIS, JÉrôme MALICK, Hristo SENDOV
}

January 29, 2014

\begin{abstract}
A set of $n \times n$ symmetric matrices whose ordered vector of eigenvalues belongs to a fixed set in $\mathbf{R}^{n}$ is called spectral or isotropic. In this paper, we establish that every locally symmetric $C^{k}$ submanifold $\mathcal{M}$ of $\mathbf{R}^{n}$ gives rise to a $C^{k}$ spectral manifold, for $k \in\{2,3, \ldots, \infty, \omega\}$. An explicit formula for the dimension of the spectral manifold in terms of the dimension and the intrinsic properties of $\mathcal{M}$ is derived. This work builds upon the results from [20] and [19], and uses characteristic properties of locally symmetric submanifolds established in [7].
\end{abstract}

Key words. spectral manifold, isotropic set, symmetric matrix, eigenvalue, locally symmetric set, invariant function.

AMS Subject Classification. Primary 15A18, 53B25 Secondary 47A45, 05A05.

\section{Contents}

1 Introduction $\quad 1$

2 Locally symmetric functions and manifolds 3

2.1 Notation and definitions . . . . . . . . . . . . . . . . . . . . . . . . . . . .

2.2 Locally symmetric manifolds . . . . . . . . . . . . . . . . . . . 5

2.3 Properties of locally symmetric manifolds . . . . . . . . . . . . . . . 8

3 Spectral manifolds $\quad 11$

3.1 Lift into $\mathbf{S}^{n}\left(\right.$ case $\left.P_{*}=\mathrm{id}_{n}\right) \ldots \ldots \ldots \ldots \ldots \ldots \ldots$

3.2 Reduction of the ambient space (general case) . . . . . . . . . . . . . . . . . . . . . . . . . . . . . . .

3.3 Transfer of the local approximation . . . . . . . . . . . . . . . 17

3.4 Transfer of local equations, proof of the main result . . . . . . . . . . . . . 21

\section{Introduction}

Let $\mathbf{R}_{\geq}^{n}$ stand for all vectors $x \in \mathbf{R}^{n}$ with $x_{1} \geq x_{2} \geq \cdots \geq x_{n}$. Denoting by $\mathbf{S}^{n}$ the Euclidean space of $n \times n$ symmetric matrices with inner product $\langle X, Y\rangle=\operatorname{tr}(X Y)$, we consider the spectral mapping $\lambda$, that is, a function from the space $\mathbf{S}^{n}$ to $\mathbf{R}^{n}$, which associates to $X \in \mathbf{S}^{n}$ the vector $\lambda(X) \in \mathbf{R}_{>}^{n}$ of its eigenvalues. More precisely, for a matrix $X \in \mathbf{S}^{n}$, the vector $\lambda(X)=\left(\lambda_{1}(X), \ldots, \lambda_{n}(X)\right)$ consists of the eigenvalues of $X$ counted with multiplicities and ordered in a non-increasing way:

$$
\lambda_{1}(X) \geq \lambda_{2}(X) \geq \cdots \geq \lambda_{n}(X)
$$


The object of study in this paper are spectral sets, that is, subsets of $\mathbf{S}^{n}$ stable under orthogonal similarity transformations: a subset $M \subset \mathbf{S}^{n}$ is a spectral set if for all $X \in M$ and $U \in \mathbf{O}^{n}$ we have $U^{\top} X U \in M$, where $\mathbf{O}^{n}$ is the group of $n \times n$ orthogonal matrices. In other words, if a matrix $X$ lies in a spectral set $M \subset \mathbf{S}^{n}$, then so does its orbit under the natural action of the group $\mathbf{O}^{n}$

$$
\mathbf{O}^{n} \cdot X:=\left\{U^{\top} X U: U \in \mathbf{O}^{n}\right\} .
$$

The spectral sets are entirely defined by their eigenvalues, and can be equivalently defined as inverse images of subsets of $\mathbf{R}^{n}$ by the spectral mapping $\lambda$, that is,

$$
\lambda^{-1}(M):=\left\{X \in \mathbf{S}^{n}: \lambda(X) \in M\right\}, \quad \text { for some } M \subset \mathbf{R}^{n} .
$$

For example, if $M$ is the Euclidean unit ball $B(0,1)$ of $\mathbf{R}^{n}$, then $\lambda^{-1}(M)$ is the Euclidean unit ball of $\mathbf{S}^{n}$ as well. A spectral set can be written as union of orbits:

$$
\lambda^{-1}(M)=\bigcup_{x \in M} \mathbf{O}^{n} \cdot \operatorname{Diag}(x)
$$

where $\operatorname{Diag}(x)$ denotes the diagonal matrix with the vector $x \in \mathbf{R}^{n}$ on the main diagonal. Notice that each orbit is an analytic submanifold of $\mathbf{S}^{n}$, see Example 2.7 for details.

In this context, a general question arises: What properties on $M$ remain true on the corresponding spectral set $\lambda^{-1}(M)$ ? In the sequel we often refer to this as the transfer principle. The spectral mapping $\lambda$ has nice geometrical properties, but it may behave very badly as far as, for example, differentiability is concerned. This imposes intrinsic difficulties for the formulation of a generic transfer principle.

Invariance properties of $M$ under permutations often correct such bad behavior and allow us to deduce transfer properties between the sets $M$ and $\lambda^{-1}(M)$. A set $M \subset \mathbf{R}^{n}$ is symmetric if $\sigma M=M$ for all permutations $\sigma$ on $n$ elements, where the permutation $\sigma$ permutes the coordinates of vectors in $\mathbf{R}^{n}$ in the natural way. Thus, if the set $M \subset \mathbf{R}^{n}$ is symmetric, then properties such as closedness and convexity are transferred between $M$ and $\lambda^{-1}(M)$. Namely, $M$ is closed (respectively, convex [14], prox-regular [5]) if and only if $\lambda^{-1}(M)$ is closed (respectively, convex, prox-regular). The next result is another interesting example of such a transfer.

Proposition 1.1 (Transferring algebraicity). Let $\mathcal{M} \subset \mathbf{R}^{n}$ be a symmetric algebraic variety. Then, $\lambda^{-1}(\mathcal{M})$ is an algebraic variety of $\mathbf{S}^{n}$.

Proof. Let $p$ be any polynomial equation of $\mathcal{M}$, that is $p(x)=0$ if and only if $x \in \mathcal{M}$. Define the symmetric polynomial $q(x):=\sum_{\sigma} p^{2}(\sigma x)$. Notice that $q$ is again a polynomial equation of $\mathcal{M}$ and $q(\lambda(X))$ is an equation of $\lambda^{-1}(\mathcal{M})$. We just have to prove that $q \circ \lambda$ is a polynomial in the entries of $X$. It is known that $q$ can be written as a polynomial of the elementary symmetric polynomials $p_{1}, p_{2}, \ldots, p_{n}$. Each $p_{j}(\lambda(X))$, up to a sign, is a coefficient of the characteristic polynomial of $X$, thus it is a polynomial in $X$. Thus, we can complete the proof.

Concurrently, similar transfer properties hold for spectral functions, that is, functions $F: \mathbf{S}^{n} \rightarrow$ $\mathbf{R}^{n}$ which are constant on the orbits $\mathbf{O}^{n} \cdot X$ or equivalently, functions $F$ that can be written as $F=f \circ \lambda$ with $f: \mathbf{R}^{n} \rightarrow \mathbf{R}$ being symmetric, that is invariant under any permutation of entries of $x$. Since $f$ is symmetric, closedness and convexity are transferred between $f$ and $F$ (see [14] for details). More surprisingly, differentiability properties are also transferred (see [1], [20], [19], [13], [15] and [18]). As established recently in [5], the same happens for a variational property called prox-regularity (we refer to [17] for the definition). 
In this work, we study the transfer of differentiable structure of a submanifold $\mathcal{M}$ of $\mathbf{R}^{n}$ to the corresponding spectral set. This gives rise to an orbit-closed set $\lambda^{-1}(\mathcal{M})$ of $\mathbf{S}^{n}$, which, in case it is a manifold, will be called spectral manifold. Such spectral manifolds often appear in engineering sciences, often as constraints in feasibility problems (for example, in the design of tight frames [21] in image processing or in the design of low-rank controller [16] in control). Given a manifold $\mathcal{M}$, the answer, however, to the question of whether or not the spectral set $\lambda^{-1}(\mathcal{M})$ is a manifold of $\mathbf{S}^{n}$ is not direct: indeed, a careful glance at (1.1) reveals that $\mathbf{O}^{n} \cdot \operatorname{Diag}(x)$ has a natural (quotient) manifold structure, but the question is how the different strata combine as $x$ moves along $\mathcal{M}$.

For functions, transferring local properties, such as differentiability, requires some symmetry, albeit not with respect to all permutations. Many properties still hold under local symmetry, that is, invariance under permutations that preserve balls centered at the point of interest. We make this more precise in Subsection 2.1.

The main goal here is to prove that local smoothness of $\mathcal{M}$ is transferred to the spectral set $\lambda^{-1}(\mathcal{M})$, whenever $\mathcal{M}$ is locally symmetric. More precisely, our aim here is

- to prove that every connected $C^{k}$ locally symmetric manifold $\mathcal{M}$ of $\mathbf{R}^{n}$ is 'lifted' to a connected $C^{k}$ manifold $\lambda^{-1}(\mathcal{M})$ of $\mathbf{S}^{n}$, for $k \in\{2,3, \ldots, \infty, \omega\}$ (where $C^{\omega}$ stands for real analytic);

- to derive a formula for the dimension of $\lambda^{-1}(\mathcal{M})$ in terms of the dimension of $\mathcal{M}$ and some characteristic properties of $\mathcal{M}$.

We established this result, back in 2009, for the cases $k=2, k=\infty$ and $k=\omega$, through a long technical proof, in the unpublished technical note [6]. With the current manuscript we provide a shorter, tractable version of the aforementioned proof, which moreover encompasses all cases $k \in\{2,3, \ldots, \infty, \omega\}$. Notation and arguments are now been simplified, and additional comments providing extra intuition have been added. We use the results from [20] and [19], and the properties of locally symmetric submanifolds of [7].

The particular case of the lift of a $C^{\infty}$ manifold is recovered in a very recent work [4] through an indirect technique based on metric projections. This technique is specific for the case $k=\infty$, and moreover, it does not provide any information on the dimension of the spectral manifold $\lambda^{-1}(\mathcal{M})$.

The main result of the current manuscript is Theorem 3.20 proving the lift of a locally symmetric $C^{k}$ manifold together with a formula of its dimension. To get this result, we use extensively differential properties of spectral functions, as well as structural properties of locally symmetric manifolds: roughly speaking, given a manifold $\mathcal{M}$ which is locally symmetric around $x \in \mathcal{M}$, the proof splits in the two following steps.

Step 1 to exhibit a simple locally symmetric affine manifold $\mathcal{D}$, see (3.8), which will be used as a domain for a locally symmetric local equation for the manifold $\mathcal{M}$ around $x$ (Lemma 3.10);

Step 2 to show that $\lambda^{-1}(\mathcal{D})$ is an analytic manifold (Theorem 3.15) and use it as a domain to build a local equation of $\lambda^{-1}(\mathcal{M})$ (c.f. (3.12)), in order to establish that this latter spectral set is a manifold (Theorem 3.20). Let us notice, however, that $\lambda^{-1}(\mathcal{D})$ is not an affine manifold in general (see comments at the end of Section 3.2).

\section{Locally symmetric functions and manifolds}

This section does not contain any new results. It introduces relevant background notation and revises material established in [7] (and previously in [6], though in a less elaborated form) concerning the structure of a locally symmetric submanifold $\mathcal{M}$ of $\mathbf{R}^{n}$. A key notion is that of a 
characteristic partition (see Section 2.3), as well as the existence of a locally symmetric reduced tangential parametrization (Theorem 2.17).

\subsection{Notation and definitions}

A partition $P$ of a finite set $N$ is a collection of non-empty, pairwise disjoint subsets of $N$ with union $N$. The elements of a partition are sometimes called blocks. The partition $\{\{i\}: i \in N\}$ is denoted by $\mathrm{id}_{N}$. The set of all partitions of $N$ is denoted by $\Pi_{N}$. The symbol $\mathbf{R}^{N}$ denotes the set of all functions from $N$ to $\mathbf{R}$. Set $\mathbb{N}_{n}:=\{1, \ldots, n\}$. When $N=\mathbb{N}_{n}$, we simply write $\Pi_{n}, \operatorname{id}_{n}$, and $\mathbf{R}^{n}$. The partition induced by $x \in \mathbf{R}^{N}$, denoted by $P_{x}$, is defined by the indexes of the equal coordinates of $x$. More precisely, for $i, j \in \mathbb{N}_{n}$ we have: $i, j$ are in the same subset of $P_{x}$ if and only if $x_{i}=x_{j}$. Given two partitions $P$ and $P^{\prime}$ of $\Pi_{N}$ we say that $P^{\prime}$ is a refinement of $P$, written $P \preceq P^{\prime}$, if every set in $P$ is a (disjoint) union of sets from $P^{\prime}$. Given a partition $P$ of $\Pi_{N}$, define the subset $\Delta_{P}$ of $\mathbf{R}^{n}$ by

$$
\Delta_{P}:=\left\{x \in \mathbf{R}^{N}: P_{x}=P\right\} .
$$

Obviously $\Delta_{P}$ is an affine manifold, not connected in general. (By affine manifold we understand an open subset of an affine subspace of a vector space.) The collection $\left\{\Delta_{P}: P \in \Pi_{N}\right\}$ is an affine stratification of $\mathbf{R}^{N}$, and for any $x \in \mathbf{R}^{N}$ there is a $\delta>0$ such that the ball $B(x, \delta)$ intersects only strata $\Delta_{P}$ with $P \succeq P_{x}$, see [7, Section 2.2]. If the partition $P \in \Pi_{N}$ is given by $P=\left\{I_{1}, \ldots, I_{m}\right\}$, then the orthogonal and bi-orthogonal spaces of $\Delta_{P}$ have the following expressions, respectively,

$$
\begin{gathered}
\Delta_{P}^{\perp}=\left\{x \in \mathbf{R}^{N}: \sum_{j \in I_{i}} x_{j}=0 \text { for all } i \in \mathbb{N}_{m}\right\}, \\
\Delta_{P}^{\perp}=\left\{x \in \mathbf{R}^{N}: x_{i}=x_{j} \text { for any } i, j \in I_{k}, k \in \mathbb{N}_{m}\right\} .
\end{gathered}
$$

Note that $\Delta_{P}^{\perp}=\overline{\Delta_{P}}$, where the latter set is the closure of $\Delta_{P}$. We have that $\Delta_{P}^{\perp} \perp=\bigcup_{P^{\prime} \preceq P} \Delta_{P^{\prime}}$.

Denote by $\Sigma^{n}$ the group of permutations over $\mathbb{N}_{n}$. This group has a natural action on $\mathbf{R}^{n}$ and $\Pi_{n}$ defined for $x \in \mathbf{R}^{n}$ by $\sigma x:=\left(x_{\sigma^{-1}(1)}, \ldots, x_{\sigma^{-1}(n)}\right)$, and for a partition $P=\left\{I_{1}, \ldots, I_{m}\right\}$ by $\sigma P:=\left\{\left\{\sigma(i): i \in I_{k}\right\}: k=1, \ldots, m\right\}$. For a vector $x \in \mathbf{R}^{n}$ and a partition $P \in \Pi_{n}$ define the subgroups of permutations

$$
\Sigma_{P}^{n}:=\left\{\sigma \in \Sigma^{n}: \sigma P=P\right\} \quad \text { and } \quad \Sigma_{x}^{n}:=\left\{\sigma \in \Sigma^{n}: \sigma x=x\right\} .
$$

Note that $\Sigma_{P_{x}}^{n}=\Sigma_{x}^{n}$ for all $x \in \mathbf{R}^{n}$.

Definition 2.1 (Locally symmetric function). A map $f$ defined on $\mathbf{R}^{n}$ is called symmetric if

$$
f(\sigma y)=f(y) \text { for all } y \in \mathbf{R}^{n} \text { and all } \sigma \in \Sigma^{n} .
$$

The function $f$ is called locally symmetric at a point $x \in \mathbf{R}^{n}$ if

$$
f(\sigma y)=f(y) \text { for all } y \text { close to } x \text { and all } \sigma \in \Sigma_{x}^{n} .
$$

Locally symmetric functions are those which are symmetric on an open ball centered at $x$, under all permutations of entries of $x$ that preserve this ball, see [7, Section 3.1]. The above property is exactly the required invariance property needed on $f$ allowing the transfer of its differentiability properties to the spectral function $f \circ \lambda$, see forthcoming Theorem 2.2. For a proof, we refer to [20] and [22]. (We point out that even though the main result in [20] is stated for symmetric functions $f$, the supporting results are stated in locally symmetric language and the argument goes unchanged in this case.) In the sequel, given a vector $x \in \mathbf{R}^{n}$, Diag $x$ denotes the diagonal matrix with the vector $x$ on the main diagonal, and diag: $\mathbf{S}^{n} \rightarrow \mathbf{R}^{n}$ denotes its adjoint operator, defined by $\operatorname{diag}(X):=\left(x_{11}, \ldots, x_{n n}\right)$ for any matrix $X=\left(x_{i j}\right)_{i, j} \in \mathbf{S}^{n}$. 
Theorem 2.2 (Derivatives of spectral functions). Suppose that $f: \mathbf{R}^{n} \rightarrow \mathbf{R}$ is locally symmetric at $x \in \mathbf{R}_{\geq}^{n}$. The function $F: \mathbf{S}^{n} \rightarrow \mathbf{R}$ defined by $F=f \circ \lambda$ is $C^{k}$ in a neighborhood of $X \in \lambda^{-1}(x)$ if and only if $f$ is $C^{k}$ in a neighborhood of $x$. Here $k \in\{1,2, \ldots, \infty, \omega\}$. In that case we have

$$
\nabla F(X)=U^{\top}(\operatorname{Diag} \nabla f(\lambda(X))) U
$$

where $U$ is any orthogonal matrix such that $X=U^{\top}(\operatorname{Diag} \lambda(X)) U$. Equivalently, for any direction $H \in \mathbf{S}^{n}$ we have

$$
\nabla F(X)[H]=\nabla f(\lambda(X)))\left[\operatorname{diag}\left(U H U^{\top}\right)\right] .
$$

The differentiability of spectral functions will be used intensively when defining local equations of spectral manifolds. Before giving the definition of spectral manifolds and locally symmetric manifolds, let us first recall the definition of submanifolds.

Definition 2.3 (Submanifold of $\mathbf{R}^{n}$ ). A nonempty set $\mathcal{M} \subset \mathbf{R}^{n}$ is a $C^{k}$ submanifold of dimension $d$ (with $d \in\{0, \ldots, n\}$ and $k \in \mathbb{N} \cup\{\omega\}$ ) if for every $x \in \mathcal{M}$, there is a neighborhood $U \subset \mathbf{R}^{n}$ of $x$ and $C^{k}$ function $\varphi: U \rightarrow \mathbf{R}^{n-d}$ with Jacobian matrix $J \varphi(x)$ of full rank, and such that for all $y \in U$ we have $y \in \mathcal{M} \Leftrightarrow \varphi(y)=0$. The map $\varphi$ is called local equation of $\mathcal{M}$ around $x$.

Definition 2.4 (Locally symmetric sets). A set $S \subseteq \mathbf{R}^{n}$ is called strongly locally symmetric if

$$
\sigma x \in S, \quad \text { for all } x \in S \text { and all } \sigma \in \Sigma_{x}^{n} \text {. }
$$

The set $S$ is called locally symmetric if for every $x \in S$ there is a $\delta>0$ such that $S \cap B(x, \delta)$ is strongly locally symmetric set.

In other words, $S$ is locally symmetric, if for every $x \in S$ there exists $\delta>0$, such that

$$
\sigma(S \cap B(x, \delta))=S \cap B(x, \delta), \quad \text { for all } \sigma \in \Sigma_{x}^{n} .
$$

In this case, observe that $S \cap B(x, \rho)$ for $\rho \leq \delta$ is a strongly locally symmetric set as well.

Example 2.5 (Trivial examples). Obviously the whole space $\mathbf{R}^{n}$ is (strongly locally) symmetric. It is also easily seen from the definition that any stratum $\Delta_{P}$ is a strongly locally symmetric affine manifold. If $x \in \Delta_{P}$ and the ball $B(x, \delta)$ is small enough so that it intersects only strata $\Delta_{P^{\prime}}$ with $P^{\prime} \succeq P$, then $B(x, \delta)$ is strongly locally symmetric.

\subsection{Locally symmetric manifolds}

In this section we recalled from [7] the formal definition of a locally symmetric manifold (submanifold of $\mathbf{R}^{n}$ ) and illustrate this notion by means of characteristic examples.

Definition 2.6 (Locally symmetric manifold). A subset $\mathcal{M}$ of $\mathbf{R}^{n}$ is said to be a (strongly) locally symmetric manifold if $\mathcal{M}$ is both a connected submanifold of $\mathbf{R}^{n}$ without boundary and a (strongly) locally symmetric set and satisfies $\mathcal{M} \cap \mathbf{R}_{\geq}^{n} \neq \emptyset$.

The above definition includes the technical assumption $\mathcal{M} \cap \mathbf{R}_{\geq}^{n} \neq \emptyset$ since the entries of the eigenvalue vector $\lambda(X)$ are non-increasing (by definition of $\lambda$ ). This assumption is not restrictive since we can always reorder the orthogonal basis of $\mathbf{R}^{n}$ to get this property fulfilled.

Our aim is to show that $\lambda^{-1}\left(\mathcal{M} \cap \mathbf{R}_{\geq}^{n}\right)$ is a manifold. This objective will be eventually accomplished by Theorem 3.20 in Section 3. We now sketch two simple approaches that could be adopted in order to prove this result and we illustrate the difficulties that appear. 
Consider the expression (1.1) for the spectral set $\lambda^{-1}(\mathcal{M})$. Even though each orbit $\mathbf{O}^{n}$. Diag $(x)$ is well-known to be analytic manifold, see forthcoming Example 2.7, there is no straightforward approach showing that the union (1.1) is also a smooth manifold. Our strategy, developed in Section 3, uses crucial properties of locally symmetric manifolds derived in [7, Section 5], namely, the existence of a locally symmetric tangential parametrization in an appropriately reduced ambient space. This is hereby used to provide a locally symmetric local equation defined in a reduced ambient space (see Section 3.2), which in turn, is used to exhibit an explicit smooth local equation for the spectral manifold $\lambda^{-1}(\mathcal{M})$ (see Sections 3.3-3.4).

Example 2.7 (The case when $\mathcal{M}=\{x\}$.). Observe that for $x \in \mathbf{R}_{>}^{n}$, we have an exact description of the stabilizer $\mathbf{O}_{\operatorname{Diag}(x)}^{n}$ of the matrix $\operatorname{Diag}(x)$. Recall that the stabilizer of a matrix $X \in \mathbf{S}^{n}$ under the action of the orthogonal group $\mathbf{O}^{n}$

$$
\mathbf{O}_{X}^{n}:=\left\{U \in \mathbf{O}^{n}: U^{\top} X U=X\right\} .
$$

Now, considering the partition $P_{x}=\left\{I_{1}, \ldots, I_{m}\right\}$ we have that $U \in \mathbf{O}_{\operatorname{Diag}(x)}^{n}$ is a block-diagonal matrix, made of matrices $U_{i} \in \mathbf{O}^{\left|I_{i}\right|}$. Conversely, every such block-diagonal matrix belongs clearly to $\mathbf{O}_{\operatorname{Diag}(x)}^{n}$. In other words, we have the identification

$$
\mathbf{O}_{\operatorname{Diag}(x)}^{n} \simeq \mathbf{O}^{\left|I_{1}\right|} \times \cdots \times \mathbf{O}^{\left|I_{m}\right|}
$$

Since $\mathbf{O}^{p}$ is a manifold of dimension $p(p-1) / 2$, we deduce that $\mathbf{O}_{\operatorname{Diag}(x)}^{n}$ is a manifold of dimension

$$
\operatorname{dim} \mathbf{O}_{\operatorname{Diag}(x)}^{n}=\sum_{i=1}^{m} \frac{\left|I_{i}\right|\left(\left|I_{i}\right|-1\right)}{2} .
$$

It is well-known that the orbit $\mathbf{O}^{n} \cdot \operatorname{Diag}(x)$ is diffeomorphic to the quotient manifold $\mathbf{O}^{n} / \mathbf{O}_{\operatorname{Diag}(x)}^{n}$. Thus, $\mathbf{O}^{n} \cdot \operatorname{Diag}(x)$ is a submanifold of $\mathbf{S}^{n}$ of dimension

$$
\begin{aligned}
\operatorname{dim} \mathbf{O}^{n} \cdot \operatorname{Diag}(x) & =\operatorname{dim} \mathbf{O}^{n}-\operatorname{dim} \mathbf{O}_{\operatorname{Diag}(x)}^{n}=\frac{n(n-1)}{2}-\sum_{i=1}^{m} \frac{\left|I_{i}\right|\left(\left|I_{i}\right|-1\right)}{2}=\frac{n^{2}-\sum_{i=1}^{m}\left|I_{i}\right|^{2}}{2} \\
& =\sum_{1 \leq i<j \leq m}\left|I_{i}\right|\left|I_{j}\right|,
\end{aligned}
$$

where we used twice the fact that $n=\sum_{i=1}^{m}\left|I_{i}\right|$.

Let us now explain how a natural approach to show that $\lambda^{-1}(\mathcal{M})$ is a manifold, using local equations, would fail. To this end, assume that the manifold $\mathcal{M}$ of dimension $d \in\{0,1, \ldots, n\}$ is described by a smooth equation $\varphi: \mathbf{R}^{n} \rightarrow \mathbf{R}^{n-d}$ around the point $x \in \mathcal{M} \cap \mathbf{R}_{\geq}^{n}$. This gives a function $\varphi \circ \lambda$ whose zeros characterize $\lambda^{-1}(\mathcal{M})$ around $X \in \lambda^{-1}(\mathcal{M})$, that is, for all $Y \in \mathbf{S}^{n}$ around $X$

$$
Y \in \lambda^{-1}(\mathcal{M}) \Longleftrightarrow \lambda(Y) \in \mathcal{M} \Longleftrightarrow \varphi(\lambda(Y))=0 .
$$

However we cannot guarantee that the function $\Phi:=\varphi \circ \lambda$ is a smooth function unless $\varphi$ is locally symmetric (since in this case Theorem 2.2 applies). But in general, local equations $\varphi: \mathbf{R}^{n} \rightarrow \mathbf{R}$ of a locally symmetric submanifold of $\mathbf{R}^{n}$ might fail to be locally symmetric, as shown by the next easy example (see [7, Example 5.5] and also [6, Example 3.8]). 
Example 2.8 (A symmetric manifold without symmetric equations). Let us consider the following symmetric (affine) submanifold of $\mathbf{R}^{2}$ of dimension one:

$$
\mathcal{M}=\left\{(x, y) \in \mathbf{R}^{2}: x=y\right\}=\Delta((12)) .
$$

The associated spectral set

$$
\lambda^{-1}(\mathcal{M})=\left\{A \in \mathbf{S}^{2}: \lambda_{1}(A)=\lambda_{2}(A)\right\}=\left\{\alpha I_{2}: \alpha \in \mathbf{R}\right\}
$$

is a submanifold of $\mathbf{S}^{2}$ around $I_{2}=\lambda^{-1}(1,1)$. It is interesting to observe that though $\lambda^{-1}(\mathcal{M})$ is a (spectral) 1-dimensional submanifold of $\mathbf{S}^{2}$, this submanifold cannot be described by a local equation that is a composition of $\lambda$ with a symmetric local equation $\varphi: \mathbf{R}^{2} \rightarrow \mathbf{R}$ of $\mathcal{M}$ around $(1,1)$. Indeed, let us assume on the contrary that such a local equation of $\mathcal{M}$ exists, that is, there exists a smooth symmetric function $\varphi: \mathbf{R}^{2} \rightarrow \mathbf{R}$ with surjective derivative $\nabla \varphi(1,1)$ which satisfies

$$
\varphi(x, y)=0 \Longleftrightarrow x=y \text {. }
$$

Consider now the two smooth paths $c_{1}: t \mapsto(t, t)$ and $c_{2}: t \mapsto(t, 2-t)$. Since $\varphi \circ c_{1}(t)=0$ we infer

$$
\nabla \varphi(1,1)(1,1)=0 .
$$

On the other hand, since $c_{2}^{\prime}(1)=(1,-1)$ is normal to $\mathcal{M}$ at $(1,1)$, and since $\varphi$ is symmetric, we deduce that the smooth function $t \mapsto\left(\varphi \circ c_{2}\right)(t)$ has a critical point at $t=1$. Thus,

$$
0=\left(\varphi \circ c_{2}\right)^{\prime}(1)=\nabla \varphi(1,1)(1,-1) .
$$

Therefore, (2.7) and (2.8) imply that $\nabla \varphi(1,1)=(0,0)$ which is a contradiction. This proves that there is no symmetric local equation $\varphi: \mathbf{R}^{2} \rightarrow \mathbf{R}$ of the symmetric manifold $\mathcal{M}$ around $(1,1)$.

We close this section by observing that the property of local symmetry introduced in Definition 2.4 is necessary and in a sense minimal. In any case, it cannot easily be relaxed as revealed by the following examples.

Example 2.9 (A manifold without symmetry). Let us consider the set

$$
\mathcal{N}=\{(t, 0): t \in(-1,1)\} \subset \mathbf{R}^{2} .
$$

We have an explicit expression of $\lambda^{-1}(\mathcal{N})$

$$
\lambda^{-1}(\mathcal{N})=\left\{\left[\begin{array}{cc}
t \cos ^{2} \theta & t(\sin 2 \theta) / 2 \\
t(\sin 2 \theta) / 2 & t \sin ^{2} \theta
\end{array}\right],\left[\begin{array}{cc}
-t \sin ^{2} \theta & t(\sin 2 \theta) / 2 \\
t(\sin 2 \theta) / 2 & -t \cos ^{2} \theta
\end{array}\right], t \geq 0\right\} .
$$

It can be proved that this lifted set is not a submanifold of $S^{2}$ since it has a sharp point at the zero matrix, as suggested by Figure 1.

Example 2.10 (A manifold without enough symmetry). Let us consider the set

$$
\mathcal{N}=\{(t, 0,-t): t \in(-\epsilon, \epsilon)\} \subset \mathbf{R}^{3}
$$

and let $x=(0,0,0) \in \mathcal{N}$. Then, $\Delta_{P_{x}}=\{(\alpha, \alpha, \alpha): \alpha \in \mathbf{R}\}$ and $\mathcal{N}$ is a smooth submanifold of $\mathbf{R}^{3}$ that is symmetric with respect to the affine set $\Delta_{P_{x}}$, but it is not locally symmetric. It can be easily proved that the set $\lambda^{-1}(\mathcal{N})$ is not a submanifold of $\mathbf{S}_{3}$ around the zero matrix. 


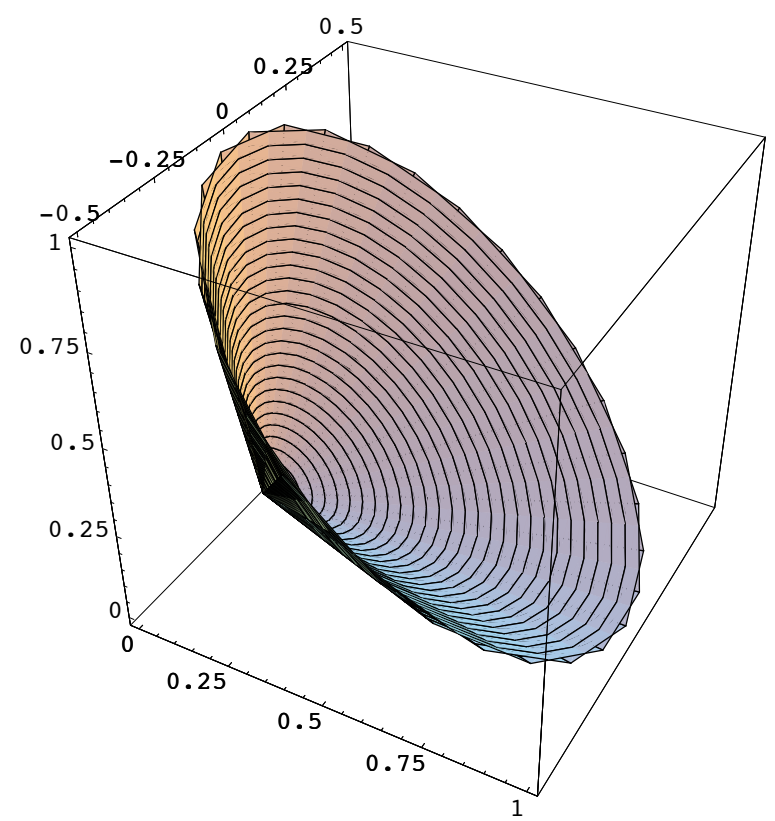

Figure 1: A spectral subset of $\mathbf{S}^{2}$ represented in $\mathbf{R}^{3}$

\subsection{Properties of locally symmetric manifolds}

In this section we collect the definitions and results from [7] that are needed in the present work. Note first that if $x \in \mathcal{M} \cap \mathbf{R}_{\geq}^{n}$, then every set in $P_{x}$ contains consecutive integers.

Definition 2.11 (Much smaller partition). Consider two partitions $P, P^{\prime} \in \Pi_{n}$.

- The partition $P^{\prime}$ is called much smaller than $P$, denoted $P^{\prime} \prec P$, whenever $P^{\prime} \prec P$ and a set in $P^{\prime}$ is formed by merging at least two sets from $P$, of which at least one contains at least two elements.

- Whenever $P^{\prime} \prec P$ but $P^{\prime}$ is not much smaller than $P$ we shall write $P^{\prime} \prec P$. In other words, if $P^{\prime} \prec P$ but $P^{\prime}$ is not much smaller than $P$, then every set in $P^{\prime}$ that is not in $P$ is formed by uniting one-element sets from $P$.

Suppose that $\mathcal{M}$ is a locally symmetric manifold. Among the partitions $P \in \Pi_{n}$ such that $\mathcal{M} \cap \mathbf{R}_{\geq}^{n} \cap \Delta_{P} \neq \emptyset$, there is a unique maximal partition $P_{*}$ called the characteristic partition of $\mathcal{M}$. The characteristic partition describes the strata that may intersect $\mathcal{M}$ :

$$
\mathcal{M} \subseteq \Delta_{P_{*}} \cup\left(\bigcup_{P \propto P_{*}} \Delta_{P}\right) \subseteq \Delta_{P_{*}}^{\perp}
$$

Formula (2.9) yields that every set in $P_{*}$ contains consecutive integers and

$$
T_{\mathcal{M}}(x) \subset \Delta_{P_{*}}^{\perp} .
$$

The stratum $\Delta_{P_{*}}$ is dense in $\mathcal{M}$ : for every $x \in \mathcal{M}$ and $\delta>0$ we have

$$
\mathcal{M} \cap \Delta_{P_{*}} \cap B(x, \delta) \neq \emptyset .
$$


Define

$$
\begin{aligned}
& \mathbb{N}_{n}^{1}:=\text { the union of all sets in } P_{*} \text { with exactly one element, and } \\
& \mathbb{N}_{n}^{2}:=\text { the union of all sets in } P_{*} \text { with more than one elements. }
\end{aligned}
$$

Clearly, $\mathbb{N}_{n}$ is the disjoint union of $\mathbb{N}_{n}^{1}$ and $\mathbb{N}_{n}^{2}$. (Either $\mathbb{N}_{n}^{1}$ or $\mathbb{N}_{n}^{2}$ may possibly be empty.)

Definition 2.12 (canonical split). The characteristic partition $P_{*}$ of $\mathcal{M}$ yields a canonical split of $\mathbf{R}^{n}$ as a direct sum of the spaces $\mathbf{R}^{\mathbb{N}_{n}^{1}}$ and $\mathbf{R}^{\mathbb{N}_{n}^{2}}$, as follows: any vector $x \in \mathbf{R}^{n}$ is represented as

$$
x=x^{F} \otimes x^{M},
$$

where

- $x^{F} \in \mathbf{R}^{\mathbb{N}_{n}^{1}}$ is the subvector of $x$ obtained by collecting the coordinates that have indices in one-element sets of $P_{*}$, preserving their relative order;

- $x^{M} \in \mathbf{R}^{\mathbb{N}_{n}^{2}}$ is the subvector of $x$ obtained by collecting the remaining coordinates, preserving their order again.

It is readily seen that the canonical split is linear and also a reversible operation. Reversibility means that given any two vectors $x^{F} \in \mathbf{R}^{\mathbb{N}_{n}^{1}}$ and $x^{M} \in \mathbf{R}^{\mathbb{N}_{n}^{2}}$, there is a unique vector $x^{F} \otimes x^{M} \in \mathbf{R}^{n}$, such that

$$
\left(x^{F} \otimes x^{M}\right)^{F}=x^{F} \quad \text { and } \quad\left(x^{F} \otimes x^{M}\right)^{M}=x^{M} .
$$

This operation is called canonical product. In the particular case $P_{*}=\mathrm{id}_{n}$ we have that $x=x^{F}$ for all $x \in \mathbf{R}^{n}$.

Definition 2.13. A partition $P \in \Pi_{n}$ is called $P_{*}$-decomposable if $P \succeq P_{\circ}$ for some $P_{\circ} \prec P_{*}$.

Note that a $P_{*}$-decomposable partition $P$ has the following property: if a set in $P$ contains elements from $\mathbb{N}_{n}^{1}$ then it cannot contain elements from $\mathbb{N}_{n}^{2}$. According to $(2.9)$, if $x \in \mathcal{M}$, then $P_{x}$ is $P_{*}$-decomposable, moreover any $P \succeq P_{x}$ is $P_{*}$-decomposable.

Definition 2.14 $\left(P_{*}\right.$-decomposition). For any $P_{*}$-decomposable partition $P$ define the partitions $P^{F} \in \Pi_{\mathbb{N}_{n}^{1}}$ and $P^{M} \in \Pi_{\mathbb{N}_{n}^{2}}$ as follows

- $P^{F}$ contains those sets of $P$ that contain only elements from $\mathbb{N}_{n}^{1}$;

- $P^{M}$ contains the remaining sets of $P$ (those containing only elements from $\mathbb{N}_{n}^{2}$ ).

The disjoint union $P=P^{F} \cup P^{M}$ is called the $P_{*}$-decomposition of $P$.

For example, applying the $P_{*}$-decomposition to $P_{*}$ yields $P_{*}^{F}=\operatorname{id}_{\mathbb{N}_{n}^{1}}$. Notice that the $P_{*^{-}}$ decomposition cannot be applied to partitions $P$ that are much smaller than $P_{*}$, since these partitions may have sets containing elements from both $\mathbb{N}_{n}^{1}$ and $\mathbb{N}_{n}^{2}$. We summarize the properties of $P_{*}$ and the $P_{*}$-decomposition that are needed later.

Proposition 2.15. (i). $P \prec P_{*}$ if and only if $P^{F} \prec \mathrm{id}_{\mathbb{N}_{n}^{1}}$ and $P^{M}=P_{*}^{M}$;

(ii). If $x \in \mathcal{M}$ and $P_{x} \preceq P$, then $P_{x}^{F} \preceq P^{F} \preceq \operatorname{id}_{\mathbb{N}_{n}^{1}}$ and $P_{*}^{M}=P_{x}^{M} \preceq P^{M}$. 
If $P \in \Pi_{n}$ is $P_{*}$-decomposable, then the partitions $P^{F} \in \Pi_{\mathbb{N}_{n}^{1}}$ and $P^{M} \in \Pi_{\mathbb{N}_{n}^{2}}$ define strata in $\mathbf{R}^{\mathbb{N}_{n}^{1}}$ and $\mathbf{R}^{\mathbb{N}_{n}^{2}}$, respectively, see (2.1), (2.2), and (2.3). A glance at formulas (2.2) and (2.3) reveals the following relations:

$$
\Delta_{P}^{\perp}=\Delta_{P^{F}}^{\perp} \otimes \Delta_{P^{M}}^{\perp} \quad \text { and } \quad \Delta_{P}^{\perp}=\Delta_{P^{F}}^{\perp} \otimes \Delta_{P^{M}}^{\perp} .
$$

Denote by $T_{\mathcal{M}}(x)$ and $N_{\mathcal{M}}(x)$ the tangent and normal spaces of $\mathcal{M}$ at a point $x \in \mathcal{M} \cap \mathbf{R}_{>}^{n}$. The local symmetry of $\mathcal{M}$ implies that these spaces are invariant under all permutations $\sigma \in \Sigma_{x}^{n}$. For any $x \in \mathcal{M}$

$$
\begin{aligned}
T_{\mathcal{M}}(x) & =\left(T_{\mathcal{M}}(x) \cap \Delta_{P_{x}}^{\perp}\right) \oplus\left(T_{\mathcal{M}}(x) \cap \Delta_{P_{x}}^{\perp}\right), \text { and } \\
N_{\mathcal{M}}(x) & =\left(N_{\mathcal{M}}(x) \cap \Delta_{P_{x}}^{\perp}\right) \oplus\left(N_{\mathcal{M}}(x) \cap \Delta_{P_{x}}^{\perp}\right) .
\end{aligned}
$$

It has been established in [7, Section 5.1] that for any $x \in \mathcal{M}$

$$
\begin{aligned}
& \text { if } w \in T_{\mathcal{M}}(x) \text { then } w^{M} \in \Delta_{P_{x}^{M}}^{\perp} \text {, and } \\
& \text { if } v \in N_{\mathcal{M}}(x) \text { then } v^{F} \in \Delta_{P_{x}^{F}}^{\perp} \text {. }
\end{aligned}
$$

Next lemma complements the structural property (2.16). It is proved in [7, Lemma 6.1].

Lemma 2.16. For every $x \in \mathcal{M}$ and $\epsilon>0$, there exists a $w \in T_{\mathcal{M}}(x) \cap B(0, \epsilon)$, such that in the vector $w^{F} \in \mathbf{R}^{\mathbb{N}_{n}^{1}}$ every subvector $w_{I}^{F}$ has distinct coordinates for every set $I$ in the partition $P_{x}^{F}$.

In the rest of this section, we briefly recall a local equation, called tangential parametrization, for a submanifold of $\mathbf{R}^{n}$, specialized to our context of a locally symmetric manifold $\mathcal{M}$.

To this end, let $\pi_{T}: \mathbf{R}^{n} \rightarrow T_{\mathcal{M}}(x)$ denote the orthogonal projection onto the tangent space at $x$ and let $\pi_{N}$ be the orthogonal projection onto the normal space $N_{\mathcal{M}}(x)$. Let $\bar{\pi}_{T}: \mathbf{R}^{n} \rightarrow x+T_{\mathcal{M}}(x)$ be the projection onto the affine space $x+T_{\mathcal{M}}(x)$, and similarly, let $\bar{\pi}_{N}$ denote the projection of $\mathbf{R}^{n}$ onto $x+N_{\mathcal{M}}(x)$. Note that for all $y \in \mathbf{R}^{n}$ sufficiently close to $x$ we have

$$
\bar{\pi}_{T}(y)+\bar{\pi}_{N}(y)=x+y .
$$

The local symmetry of $\mathcal{M}$ implies the existence of $\delta>0$ such that

$$
\sigma \bar{\pi}_{T}(y)=\bar{\pi}_{T}(\sigma y) \quad \text { and } \quad \sigma \bar{\pi}_{N}(y)=\bar{\pi}_{N}(\sigma y)
$$

for all $y \in B(x, \delta)$ and all $\sigma \in \Sigma_{x}^{n}$. Shrinking further $\delta>0$ we can ensure that the following conditions hold.

(A1) The restriction $\bar{\pi}_{T}: \mathcal{M} \cap B(x, \delta) \rightarrow x+T_{\mathcal{M}}(x)$ is a diffeomorphism onto its image.

(A2) The ball $B(x, \delta)$ intersects only strata $\Delta_{P}$ with $P \succeq P_{x}$.

Under the above conditions $\mathcal{M} \cap B(x, \delta)$ is a strongly locally symmetric manifold (see Equation (2.5)). In addition, there exists a smooth map

$$
\phi:\left(x+T_{\mathcal{M}}(x)\right) \cap B(x, \delta) \rightarrow N_{\mathcal{M}}(x),
$$

such that

$$
\mathcal{M} \cap B(x, \delta)=\left\{y+\phi(y) \in \mathbf{R}^{n}: y \in\left(x+T_{\mathcal{M}}(x)\right) \cap B(x, \delta)\right\} .
$$

In words, the function $\phi$ measures the difference between the manifold and its tangent space. Clearly, $\phi \equiv 0$ if $\mathcal{M}$ is an affine manifold around $x$. Note that, technically, the domain of the 
map $\phi$ is the (strongly symmetric) open set $\bar{\pi}_{T}(\mathcal{M} \cap B(x, \delta)$ ), which may be a proper subset of $\left(x+T_{\mathcal{M}}(x)\right) \cap B(x, \delta)$. For sake of readability we shall not introduce the more precise (but also more complicated notation) of a rectangular neighborhoods around $x$.

We say that the map $\psi:\left(x+T_{\mathcal{M}}(x)\right) \cap B(x, \delta) \rightarrow \mathcal{M} \cap B(x, \delta)$ defined by

$$
\psi(y)=y+\phi(y)
$$

is the tangential parametrization of $\mathcal{M}$ around $x$. This function is indeed smooth, one-to-one and onto, with a full rank Jacobian matrix $J \psi(x)$ : it is a local diffeomorphism at $x$, and more precisely its inverse is $\bar{\pi}_{T}$, that is, locally $\bar{\pi}_{T}(\psi(y))=y$. We are now ready to state the main result of [7] (namely, [7, Theorem 5.4]). We point out that the proof of (2.23) utilizes the fundamental relation (2.17) established in [7, Theorem 5.1].

Theorem 2.17 (Tangential parametrization). Let $x \in \mathcal{M}$. Then, the function $\phi$ in the tangential parametrization satisfies

$$
\phi(x) \in N_{\mathcal{M}}(x) \cap \Delta_{P_{x}}^{\perp \perp} .
$$

Moreover, for all $y \in\left(x+T_{\mathcal{M}}(x)\right) \cap B(x, \delta)$ and for all $\sigma \in \Sigma_{x}^{n}$ we have

$$
\psi(\sigma y)=\sigma \psi(y)
$$

and

$$
\phi(\sigma y)=\sigma \phi(y)=\phi(y)
$$

\section{Spectral manifolds}

We begin this section with an example of the special case when the (locally symmetric) manifold $\mathcal{M}$ is (a relatively open subset of) a stratum $\Delta_{P}$. In this case, basic algebraic arguments allow to conclude directly that $\lambda^{-1}(\mathcal{M})$ is a smooth manifold.

Example 3.1 (Lift of stratum $\Delta_{P}$ ). Suppose that the manifold $\mathcal{M}$ is (a relatively open subset of) a stratum $\Delta_{P}$ and intersects $\mathbf{R}_{\geq}^{n}$. In this case, we show directly that the spectral set $\lambda^{-1}(\mathcal{M})$ is an analytic (fiber) manifold using basic arguments exposed in Example 2.7. As stated therein, the orbit $\mathbf{O}_{\operatorname{Diag}(x)}^{n}$ is a submanifold of $\mathbf{S}^{n}$ of dimension

$$
\sum_{1 \leq i<j \leq m}\left|I_{i}\right|\left|I_{j}\right|
$$

where $P=\left\{I_{1}, \ldots, I_{m}\right\}$. The key is to observe that, in our case, for any $x \in \mathcal{M}$ we have

$$
\mathbf{O}_{\operatorname{Diag}(x)}^{n} \simeq \mathbf{O}^{\left|I_{1}\right|} \times \cdots \times \mathbf{O}^{\left|I_{m}\right|}
$$

and $P_{x}=P$. Then, all the orbits $\mathbf{O}^{n} \cdot \operatorname{Diag}(x)$ are manifolds diffeomorphic to $\mathbf{O}^{n} / \mathbf{O}_{\operatorname{Diag}(\bar{x})}^{n}$ (fibers), whence of the same dimension. We deduce that $\lambda^{-1}(\mathcal{M})$ is a submanifold of $\mathbf{S}^{n}$ diffeomorphic to the direct product $\mathcal{M} \times\left(\mathbf{O}^{n} / \mathbf{O}_{\operatorname{Diag}(\bar{x})}^{n}\right)$, with dimension

$$
\operatorname{dim} \lambda^{-1}(\mathcal{M})=d+\sum_{1 \leq i<j \leq m}\left|I_{i}\right|\left|I_{j}\right| .
$$

The example is complete. 
The proof of the general situation (that is, $\mathcal{M}$ arbitrary locally symmetric manifold) is a generalization of the above arguments, albeit a nontrivial one. The strategy is more precisely explained in Section 3.2. Before this, in Section 3.1 we treat the special case $P_{*}=\mathrm{id}_{n}$. In this case, the proof that a locally symmetric manifold lifts smoothly in $\mathbf{S}^{n}$ runs out without extra technicalities, illustrating the main ideas. In addition, the main result in the special case $P_{*}=\mathrm{id}_{n}$ is a needed step in the general case.

Before we proceed, let us introduce the following definition.

Definition 3.2 (Ordered partition). A partition $P=\left\{I_{1}, \ldots, I_{m}\right\}$ of $\mathbb{N}_{n}$ is called ordered if for any $1 \leq i<j \leq m$ the smallest element in $I_{i}$ is (strictly) smaller than the smallest element in $I_{j}$.

For example, the ordered version of the partition $\{\{4\},\{3,2\},\{1,5\}\}$ of $\mathbb{N}_{5}$ is $\{\{1,5\},\{2,3\},\{4\}\}$. Consider an ordered partition $P=\left\{I_{1}, \ldots, I_{m}\right\}$ of $\mathbb{N}_{n}$. Consider the space $\mathbf{S}_{P}^{n}$ of all blockdiagonal symmetric matrices in which the $\ell$-th block is of size $\left|I_{\ell}\right|$, and denote by $\mathbf{O}_{P}^{n}$ the subgroup of block-diagonal orthogonal matrices in which the $\ell$-th block is of size $\left|I_{\ell}\right|$. Denote by $X_{P}=\operatorname{Diag}\left(X_{1}, \ldots, X_{m}\right)$ an element of $\mathbf{S}_{P}^{n}$, where $X_{\ell} \in \mathbf{S}^{\left|I_{\ell}\right|}$. For any $X_{P} \in \mathbf{S}_{P}^{n}$, we define

$$
\lambda_{P}\left(X_{P}\right):=\left(\lambda\left(X_{1}\right), \ldots, \lambda\left(X_{m}\right)\right) \in \mathbf{R}^{n} .
$$

Note the difference between $\lambda_{P}\left(X_{P}\right)$ and $\lambda\left(X_{P}\right)$ : the coordinates of the vector $\lambda_{P}\left(X_{P}\right)$ are ordered within each block, while those of $\lambda\left(X_{P}\right)$ are ordered globally. For technical reasons we shall need a slight modification of Theorem 2.2 (Derivatives of spectral functions) to cover the case of spectral functions of the type $f \circ \lambda_{P}$ on $\mathbf{S}_{P}^{n}$.

Lemma 3.3. Suppose that $f: \mathbf{R}^{n} \rightarrow \mathbf{R}$ is locally symmetric at $x \in \mathbf{R}_{\geq}^{n}$ and let $\delta>0$ be small enough. The function $F: \mathbf{S}_{P_{x}}^{n} \rightarrow \mathbf{R}$ defined by $F=f \circ \lambda_{P_{x}}$ is $C^{k}$ on $\lambda_{P_{x}}^{-1}(B(x, \delta))$ if and only if $f$ is $C^{k}$ on $B(x, \delta)$. The Jacobian of $f \circ \lambda_{P_{x}}$ at $X \in \lambda_{P_{x}}^{-1}(B(x, \delta))$ applied to $H \in \mathbf{S}_{P_{x}}^{n}$ is

$$
J\left(f \circ \lambda_{P_{x}}\right)(X)[H]=J(f \circ \lambda)(X)[H] .
$$

Here $k \in\{1,2,3, \ldots, \infty, \omega\}$.

Proof. Let $P_{x}=\left\{I_{1}, \ldots, I_{m}\right\}$ and let $X=\operatorname{Diag}\left(X_{1}, \ldots, X_{m}\right) \in \lambda_{P_{x}}^{-1}(B(x, \delta))$. Suppose $B(x, \delta)$ intersects only strata $\Delta_{P}$ with $P \succeq P_{x}$. The fact that $x \in \mathbf{R}_{>}^{n}$ implies that $\lambda_{\min }\left(X_{\ell}\right)>\lambda_{\max }\left(X_{\ell+1}\right)$ for $1 \leq \ell \leq m-1$. Hence, $\lambda_{P_{x}}(X)=\lambda(X)$ and the claim follows from Theorem 2.2.

\subsection{Lift into $\mathrm{S}^{n}\left(\right.$ case $\left.P_{*}=\mathrm{id}_{n}\right)$}

In this section, we consider the case when $P_{*}=\mathrm{id}_{n}$. This condition implies that $P^{F}=P$ for any $P_{*}$-decomposable partition $P$ and $v^{F}=v$ for any $v \in \mathbf{R}^{n}$. Thus, Property (2.17) simplifies to

$$
N_{\mathcal{M}}(x) \subseteq \Delta_{P_{x}}^{\perp} \quad \text { for all } x \in \mathcal{M} .
$$

The goal here is to establish, under conditions (A1)-(A2), that if $x \in \mathcal{M} \cap \mathbf{R}_{\geq}^{n}$ the set $\lambda_{P_{x}}^{-1}(\mathcal{M} \cap$ $B(x, \delta))$ is a submanifold of $\mathbf{S}_{P_{x}}^{n}$, and to calculate its dimension. This is an intermediate step on the way to prove that $\lambda^{-1}(\mathcal{M})$ is a submanifold of $\mathbf{S}^{n}$. The benefit of treating first the special case $P_{*}=\mathrm{id}_{n}$ is two fold. On the one hand the results in this subsection are needed later and on the other hand the succession of arguments in the general case is similar to the one here.

Using (3.2), we can exhibit easily a locally symmetric local equation of $\mathcal{M}$. Thus, fix $x \in \mathcal{M} \cap \mathbf{R}_{\geq}^{n}$ and recall the definitions of the projections $\bar{\pi}_{T}$ and $\bar{\pi}_{N}$. 
Lemma 3.4. If $P_{*}=\mathrm{id}_{n}$ then $\bar{\pi}_{N}$ is locally symmetric at $x$.

Proof. Take any $y \in B\left(x, \delta^{\prime}\right)$, where $\delta>\delta^{\prime}>0$ is small so that $B(x, \delta)$ intersects only strata $\Delta_{P}$ for $P \succeq P_{x}$. Without loss of generality, there is $z \in \mathcal{M} \cap B(x, \delta)$ such that $\bar{\pi}_{N}(y)=\bar{\pi}_{N}(z)$. The fact that $z \in \Delta_{P_{z}}^{\perp}$ together with (3.2), applied to $z$ gives that $z+N_{\mathcal{M}}(z) \subseteq \Delta_{P_{z}}^{\perp}$. Therefore $\bar{\pi}_{N}(z) \in \Delta_{P_{z}}^{\perp}$, and consequently for all $\sigma \in \Sigma_{x}^{n}$, we have $\bar{\pi}_{N}(\sigma y)=\sigma \bar{\pi}_{N}(y)=\sigma \bar{\pi}_{N}(z)=\bar{\pi}_{N}(z)=\bar{\pi}_{N}(y)$. This means that $\bar{\pi}_{N}$ is locally symmetric at $x$.

Recall also the definition of $\phi$ given by (2.20) and the conditions on the ball $B(x, \delta)$ there. Define the function

$$
\bar{\phi}:\left\{\begin{aligned}
B(x, \delta) & \longrightarrow N_{\mathcal{M}}(x) \\
y & \longmapsto x+\phi\left(\bar{\pi}_{T}(y)\right)-\bar{\pi}_{N}(y) .
\end{aligned}\right.
$$

Lemma 3.5 (Existence of a locally symmetric local equation in the case $P_{*}=\mathrm{id}_{n}$ ). The function $\bar{\phi}$ defined by (3.3) is a local equation of $\mathcal{M}$ around $x \in \mathcal{M}$ that is locally symmetric. In other words

$$
\bar{\phi}(\sigma y)=\sigma \bar{\phi}(y)=\bar{\phi}(y) \quad \text { for all } y \in B(x, \delta) \text { and all } \sigma \in \Sigma_{x}^{n} .
$$

Proof. For $y \in B(x, \delta)$ we have that

$$
\bar{\phi}(y)=0 \Longleftrightarrow \bar{\pi}_{N}(y)=x+\phi\left(\bar{\pi}_{T}(y)\right) \Longleftrightarrow y=\bar{\pi}_{T}(y)+\phi\left(\bar{\pi}_{T}(y)\right) \Longleftrightarrow y \in \mathcal{M} \cap B(x, \delta),
$$

using successively (2.18) and (2.21). The Jacobian mapping $J \bar{\phi}(y)$ is a linear map from $\mathbf{R}^{n}$ to $N_{\mathcal{M}}(x)$, which, when applied to any direction $h$, yields

$$
J \bar{\phi}(y)[h]=J \phi\left(\bar{\pi}_{T}(y)\right)\left[\pi_{T}(h)\right]-\pi_{N}(h) .
$$

Clearly, for $h \in N_{\mathcal{M}}(x)$ we have $J \bar{\phi}(x)[h]=-h$ showing that the Jacobian in onto and hence of full rank. Thus, $\bar{\phi}$ is a local equation of $\mathcal{M}$ around $x$. In view of Theorem 2.17 and the symmetries of the projections, for any $\sigma \in \Sigma_{x}^{n}$ and any $y \in B(x, \delta)$ we have $\left(\phi \circ \bar{\pi}_{T}\right)(\sigma y)=\left(\phi \circ \bar{\pi}_{T}\right)(y)$. This, implies that

$$
\sigma^{-1} \bar{\phi}(\sigma y)=\sigma^{-1}\left(x+\left(\phi \circ \bar{\pi}_{T}\right)(y)-\sigma \bar{\pi}_{N}(y)\right)=\bar{\phi}(y) .
$$

Since $\bar{\phi}(y) \in N_{\mathcal{M}}(x) \subset \Delta_{P_{x}}^{\perp}$, we obtain the second claimed equality $\sigma \bar{\phi}(y)=\bar{\phi}(y)$.

Next, consider the map

$$
\bar{\Phi}:\left\{\begin{aligned}
\lambda_{P_{x}}^{-1}(B(x, \delta)) & \longrightarrow N_{\mathcal{M}}(x) \\
X & \longmapsto\left(\bar{\phi} \circ \lambda_{P_{x}}\right)(X)=x+\phi\left(\bar{\pi}_{T}\left(\lambda_{P_{x}}(X)\right)\right)-\bar{\pi}_{N}\left(\lambda_{P_{x}}(X)\right) .
\end{aligned}\right.
$$

Since $\bar{\phi}$ is a local equation of $\mathcal{M}$ around $x$, we deduce that for $X \in \mathbf{S}_{P_{x}}^{n}$

$$
X \in \lambda_{P_{x}}^{-1}(\mathcal{M} \cap B(x, \delta)) \Longleftrightarrow \lambda_{P_{x}}(X) \in \mathcal{M} \cap B(x, \delta) \Longleftrightarrow \bar{\Phi}(X)=0 .
$$

Thus, in order to prove that $\bar{\Phi}$ is a local equation for $\lambda_{P_{x}}^{-1}(\mathcal{M} \cap B(x, \delta))$, it remains to establish that $\bar{\Phi}$ is $C^{k}$-differentiable and that its Jacobian $J \bar{\Phi}$ has full rank at $X \in \lambda_{P_{x}}^{-1}(x)$. This is accomplished in Theorem 3.7. First we need the following lemma.

Lemma 3.6. The function $\bar{\pi}_{N} \circ \lambda_{P_{x}}$ is analytic on $\lambda_{P_{x}}^{-1}(B(x, \delta))$. Moreover, at any $X \in \lambda_{P_{x}}^{-1}(B(x, \delta))$ and any direction $H \in \mathbf{S}_{P_{x}}^{n}$ we have

$$
J\left(\bar{\pi}_{N} \circ \lambda_{P_{x}}\right)(X)[H]=\pi_{N}\left(\operatorname{diag}\left(U H U^{\top}\right)\right),
$$

where $U \in \mathbf{O}_{P_{x}}^{n}$ is such that $X=U^{\top}\left(\operatorname{Diag} \lambda_{P_{x}}(X)\right) U$. 
Proof. By Lemma 3.4, $\bar{\pi}_{N}$ is locally symmetric at $x$. By Lemma 3.3, $\bar{\pi}_{N} \circ \lambda_{P_{x}}$ is analytic on $\lambda_{P_{x}}^{-1}(B(x, \delta))$. Its Jacobian at $X \in \lambda_{P_{x}}^{-1}(B(x, \delta))$ in the direction $H \in \mathbf{S}_{P_{x}}^{n}$ is

$$
J\left(\bar{\pi}_{N} \circ \lambda_{P_{x}}\right)(X)[H]=J\left(\bar{\pi}_{N} \circ \lambda\right)(X)[H]=J \bar{\pi}_{N}(\lambda(X))\left[\operatorname{diag}\left(U H U^{\top}\right)\right]=\pi_{N}\left(\operatorname{diag}\left(U H U^{\top}\right)\right),
$$

where the second equality following by (2.4).

Theorem 3.7 (Main result $\left(\right.$ case $\left.P_{*}=\mathrm{id}_{n}\right)$ ). Let $\mathcal{M}$ be a locally symmetric $C^{k}$ submanifold of $\mathbf{R}^{n}$ of dimension d. Suppose $P_{*}=\mathrm{id}_{n}$, fix $x \in \mathcal{M} \cap \mathbf{R}_{\perp}^{n}$ and let $\delta>0$ be such that conditions (A1)-(A2) hold. Then, $\lambda^{-1}(\mathcal{M} \cap B(x, \delta))$ is a $C^{k}$ submanifold of $\mathbf{S}^{n}$ with codimension $n-d$. Here $k \in\{2,3, \ldots, \infty, \omega\}$.

Proof. By Theorem 2.17 and (2.19), the function $\phi \circ \bar{\pi}_{T}$ is locally symmetric at $x$. Therefore Lemma 3.3 yields that $\phi \circ \bar{\pi}_{T} \circ \lambda_{P_{x}}$ is $C^{k}$ on $\lambda_{P_{x}}^{-1}(B(x, \delta))=\lambda^{-1}(B(x, \delta))$. Combining this with Lemma 3.6, we deduce that the function $\bar{\Phi}$ defined by $(3.4)$ is $C^{k}$ on $\lambda^{-1}(B(x, \delta))$.

Let us now show that the Jacobian $J \bar{\Phi}$ has full rank at $X \in \lambda^{-1}(B(x, \delta))$. First, the gradient of the $i$-th coordinate function $\phi_{i} \circ \bar{\pi}_{T}$ at $x$ applied to the direction $h$ is

$$
\nabla\left(\phi_{i} \circ \bar{\pi}_{T}\right)(x)[h]=\nabla \phi_{i}\left(\bar{\pi}_{T}(x)\right)\left[\pi_{T}(h)\right] .
$$

Second, Lemma 3.3 and Theorem 2.2 give that the gradient of $\phi_{i} \circ \bar{\pi}_{T} \circ \lambda$ at $X$ in the direction $H \in \mathbf{S}^{n}$ is

$$
\nabla\left(\phi_{i} \circ \bar{\pi}_{T} \circ \lambda\right)(X)[H]=\nabla \phi_{i}\left(\bar{\pi}_{T}(\lambda(X))\right)\left[\pi_{T}\left(\operatorname{diag}\left(U H U^{\top}\right)\right)\right],
$$

where $U \in \mathbf{O}^{n}$ is such that $X=U^{\top}(\operatorname{Diag} \lambda(X)) U$. Combining this with Lemma 3.6 we obtain the following expression for the derivative of the map $\bar{\Phi}$ :

$$
J \bar{\Phi}(X)[H]=J \phi\left(\bar{\pi}_{T}(\lambda(X))\right)\left[\pi_{T}\left(\operatorname{diag}\left(U H U^{\top}\right)\right)\right]-\pi_{N}\left(\operatorname{diag}\left(U H U^{\top}\right)\right) .
$$

Notice that for any $h \in N_{\mathcal{M}}(x)$ defining $H:=U^{\top}(\operatorname{Diag} h) U \in \mathbf{S}^{n}$ we have

$$
J \bar{\Phi}(X)[H]=-h,
$$

showing that the linear map $J \bar{\Phi}(X): \mathbf{S}^{n} \rightarrow N_{\mathcal{M}}(x)$ is onto and thus has full rank. In view of (3.5), $\bar{\Phi}$ is a local equation of $\mathcal{M}$ around $X$.

Since $d=\operatorname{dim}(\mathcal{M})=\operatorname{dim}\left(T_{\mathcal{M}}(x)\right)$ and $\operatorname{dim}\left(N_{\mathcal{M}}(x)\right)=n-d$ and since $\bar{\phi}$ and $\bar{\Phi}$ are local equations of the manifolds $\mathcal{M}$ and $\lambda^{-1}(\mathcal{M} \cap B(x, \delta))$ respectively, these manifolds have the same codimension $n-d$.

\subsection{Reduction of the ambient space (general case)}

We now consider a manifold $\mathcal{M}$ with general characteristic partition $P_{*}$ and $\delta>0$ such that conditions (A1)-(A2) hold. Using (2.21) and (2.23) we obtain the inclusion

$$
\mathcal{M} \cap B(x, \delta) \subset\left(x+T_{\mathcal{M}}(x) \oplus\left(N_{\mathcal{M}}(x) \cap \Delta_{P_{x}^{\perp}}^{\perp}\right)\right) \cap B(x, \delta) .
$$

To define a local equation of $\mathcal{M}$ in the appropriate space, we introduced the reduced tangent and normal spaces.

$$
T_{\mathcal{M}}^{\mathrm{red}}(x):=T_{\mathcal{M}}(x) \cap \Delta_{P_{x}}^{\perp} \quad \text { and } \quad N_{\mathcal{M}}^{\mathrm{red}}(x):=N_{\mathcal{M}}(x) \cap \Delta_{P_{x}}^{\perp} .
$$


Note that theses spaces are invariant under permutations $\sigma \in \Sigma_{x}^{n}$. For later use when calculating the dimension of spectral manifolds, we denote

$$
n^{\mathrm{red}}:=\operatorname{dim} N_{\mathcal{M}}^{\mathrm{red}}(x)
$$

We now describe the set on which the local equation of $\lambda^{-1}(\mathcal{M})$ will be defined. Let $x=x^{F} \otimes x^{M}$ be the canonical split of $x$ in $\mathbf{R}^{n}$. Naturally $B\left(x^{F}, \delta_{1}\right)$ denotes the open ball in $\mathbf{R}^{\mathbb{N}_{n}^{1}}$ centered at $x^{F}$ with radius $\delta_{1}$, and $B\left(x^{M}, \delta_{2}\right)$ denotes the open ball in $\mathbf{R}^{\mathbb{N}_{n}^{2}}$ centered at $x^{M}$ with radius $\delta_{2}$. Define the following rectangular neighborhood of $x$

$$
B\left(x, \delta_{1}, \delta_{2}\right):=B\left(x^{F}, \delta_{1}\right) \otimes B\left(x^{M}, \delta_{2}\right) .
$$

Choose $\delta_{1}, \delta_{2}$ so that $B\left(x, \delta_{1}, \delta_{2}\right) \subset B(x, \delta)$. By conditions (A1)-(A2) and Proposition 2.15 (ii), the ball $B\left(x^{F}, \delta_{1}\right)$ intersects only strata $\Delta_{P_{F}} \subset \mathbf{R}^{\mathbb{N}_{n}^{1}}$ for $P^{F} \succeq P_{x}^{F}$, and similarly for the ball $B\left(x^{M}, \delta_{2}\right)$. Thus, $B\left(x, \delta_{1}, \delta_{2}\right)$ is invariant under permutations $\sigma \in \Sigma_{x}^{n}$. The key element in our next development is going to be the set

$$
\mathcal{D}:=\left(x+T_{\mathcal{M}}(x) \oplus N_{\mathcal{M}}^{\mathrm{red}}(x)\right) \cap B\left(x, \delta_{1}, \delta_{2}\right),
$$

which will play the role of a new ambient space. Indeed, $\mathcal{D}$ is an affine manifold of $\mathbf{R}^{n}$ and will be the domain of a symmetric local equation of $\mathcal{M}$. We gather properties of $\mathcal{D}$ in the next proposition.

Proposition 3.8 (Properties of $\mathcal{D}$ ). In the situation above, there holds

$$
T_{\mathcal{M}}(x) \oplus N_{\mathcal{M}}^{\mathrm{red}}(x)=T_{\mathcal{M}}^{\mathrm{red}}(x) \oplus \Delta_{P_{x}}^{\perp} .
$$

Hence, we can reformulate

$$
\mathcal{D}=\left(x+\left(T_{\mathcal{M}}^{\mathrm{red}}(x) \oplus \Delta_{P_{x}}^{\perp}\right)\right) \cap B\left(x, \delta_{1}, \delta_{2}\right) .
$$

Moreover, the set $\mathcal{D}$ is invariant under all permutations $\sigma \in \Sigma_{x}^{n}$, and hence it is a locally symmetric set.

Proof. Indeed, applying successively (3.6), (2.14) and since $T_{\mathcal{M}}(x)$ and $N_{\mathcal{M}}(x)$ are orthogonal complements, we have

$$
\begin{aligned}
T_{\mathcal{M}}(x) \oplus N_{\mathcal{M}}^{\mathrm{red}}(x) & =T_{\mathcal{M}}(x) \oplus\left(N_{\mathcal{M}}(x) \cap \Delta_{P_{x}}^{\perp}\right) \\
& =\left(T_{\mathcal{M}}(x) \cap \Delta_{P_{x}}^{\perp}\right) \oplus\left(T_{\mathcal{M}}(x) \cap \Delta_{P_{x}}^{\perp}\right) \oplus\left(N_{\mathcal{M}}(x) \cap \Delta_{P_{x}}^{\perp}\right) \\
& =\left(T_{\mathcal{M}}^{\perp}(x) \cap \Delta_{P_{x}}^{\perp}\right) \oplus \Delta_{P_{x}}^{\perp} \\
& =T_{\mathcal{M}}^{\mathrm{red}}(x) \oplus \Delta_{P_{x}^{\perp}}^{\perp}
\end{aligned}
$$

which yields (3.9) since $x \in \Delta_{P_{x}}^{\perp}$ and $0 \in T_{\mathcal{M}}^{\text {red }}(x)$. The invariance of $\mathcal{D}$ follows from the invariance of each set in the intersection.

Let $\bar{\pi}_{N}^{\mathrm{red}}$ and $\pi_{N}^{\mathrm{red}}$ be the projections onto $x+N_{\mathcal{M}}^{\mathrm{red}}(x)$ and $N_{\mathcal{M}}^{\mathrm{red}}(x)$ respectively. Note that

$$
x+y=\bar{\pi}_{T}(y)+\bar{\pi}_{N}^{\mathrm{red}}(y) \quad \text { for all } y \in x+T_{\mathcal{M}}(x) \oplus N_{\mathcal{M}}^{\mathrm{red}}(x) .
$$

The next result is the analogue of Lemma 3.4.

Lemma 3.9 (Local symmetry of $\bar{\pi}_{N}^{\mathrm{red}}$ ). The projection $\bar{\pi}_{N}^{\mathrm{red}}$ is locally symmetric at $x$. 
Proof. Projecting onto $x+N_{\mathcal{M}}^{\text {red }}(x)$ can be accomplished in two steps. First projecting onto $x+\Delta_{P_{x}}^{\perp}$ and then onto $x+N_{\mathcal{M}}^{\mathrm{red}}(x)$. Now, the projection onto $x+\Delta_{P_{x}}^{\perp}$ is given by $y \mapsto x+\frac{1}{\left|\Sigma_{x}^{n}\right|} \sum_{\sigma \in \Sigma_{x}^{n}} \sigma y$, see [7, Lemma 2.9]. Since it is locally symmetric at $x$, the result follows.

Similarly to (3.3) we define the map

$$
\bar{\phi}:\left\{\begin{array}{l}
\mathcal{D} \longrightarrow N_{\mathcal{M}}^{\mathrm{red}}(x) \\
y \longmapsto x+\phi\left(\bar{\pi}_{T}(y)\right)-\bar{\pi}_{N}^{\mathrm{red}}(y) .
\end{array}\right.
$$

The next lemma is an analogue of Lemma 3.5.

Lemma 3.10 (Existence of a locally symmetric local equation). The map $\bar{\phi}$ is well-defined, locally symmetric, and is a local equation of $\mathcal{M}$ around $x$.

Proof. The set $\mathcal{D}$ is chosen so that $\phi$ is well-defined. Thanks to (2.23) and the fact that $x-\bar{\pi}_{N}^{\mathrm{red}}(y) \in N_{\mathcal{M}}^{\mathrm{red}}(x)$, the range of $\bar{\phi}$ is in $N_{\mathcal{M}}^{\mathrm{red}}(x)$. The remainder of the proof follows closely that of Lemma 3.5. For all $y \in \mathcal{D}$, in view of (3.10), (2.21) and Theorem 2.17 we obtain

$$
\bar{\phi}(y)=0 \Longleftrightarrow \bar{\pi}_{N}^{\mathrm{red}}(y)=x+\phi\left(\bar{\pi}_{T}(y)\right) \Longleftrightarrow y=\bar{\pi}_{T}(y)+\phi\left(\bar{\pi}_{T}(y)\right) \Longleftrightarrow y \in \mathcal{M} \cap B(x, \delta) .
$$

The Jacobian of $\bar{\phi}$ at $y$ is a linear map from $T_{\mathcal{M}}(x) \oplus N_{\mathcal{M}}^{\mathrm{red}}(x)$ to $N_{\mathcal{M}}^{\mathrm{red}}(x)$, given by

$$
J \bar{\phi}(y)[h]=J \phi\left(\bar{\pi}_{T}(y)\right)\left[\pi_{T}(h)\right]-\pi_{N}^{\mathrm{red}}(h) .
$$

Clearly, for $h \in N_{\mathcal{M}}^{\mathrm{red}}(x)$ we have $J \bar{\phi}(x)[h]=-h$ showing that the Jacobian $J \bar{\phi}$ at $x$ is onto and has a full rank. Thus, $\bar{\phi}$ is a local equation of $\mathcal{M}$ around $x$. Finally, for any $\sigma \in \Sigma_{x}^{n}$ and any $y \in \mathcal{D}$, Theorem 2.17 shows that $\phi\left(\bar{\pi}_{T}(\sigma y)\right)=\phi\left(\sigma \bar{\pi}_{T}(y)\right)=\phi\left(\bar{\pi}_{T}(y)\right)$. Together with Lemma 3.9, the local symmetry of $\bar{\phi}$ follows.

We introduce the spectral function $\bar{\Phi}$ associated with $\bar{\phi}$

$$
\bar{\Phi}:\left\{\begin{aligned}
\lambda^{-1}(\mathcal{D}) & \longrightarrow N_{\mathcal{M}}^{\mathrm{red}}(x) \\
X & \longmapsto(\bar{\phi} \circ \lambda)(X)=x+\phi\left(\bar{\pi}_{T}(\lambda(X))\right)-\bar{\pi}_{N}^{\mathrm{red}}(\lambda(X)) .
\end{aligned}\right.
$$

By construction, we get that the zeros of $\bar{\Phi}$ characterize $\mathcal{M}$, since

$$
X \in \lambda^{-1}(\mathcal{M} \cap B(x, \delta)) \Longleftrightarrow \lambda(X) \in \mathcal{M} \cap B(x, \delta) \Longleftrightarrow \bar{\Phi}(X)=0 .
$$

At this stage, let us compare (3.12) with (3.4) and the particular treatment in Section 3.1. In Section 3.1 we had $N_{\mathcal{M}}(x) \subseteq \Delta_{P_{x}}^{\perp}$ yielding $N_{\mathcal{M}}^{\text {red }}(x)=N_{\mathcal{M}}(x)$ and thus $\mathcal{D}=B\left(x, \delta_{1}, \delta_{2}\right)$, an open subset of $\mathbf{R}^{n}$. Unfortunately, in the general case, there is an extra difficulty, which stems from the fact that $\mathcal{D}$ is not open in $\mathbf{R}^{n}$ and consequently the function $\bar{\Phi}$ is defined on a subset $\lambda^{-1}(\mathcal{D})$ of $\mathbf{S}^{n}$, of lower dimension. For this reason, the plan of action is as follows.

1. Transfer of local approximation. Show that the set $\lambda^{-1}(\mathcal{D})$ is an analytic manifold locally around $X \in \lambda^{-1}(x)$ and calculate its dimension;

2. Transfer of local equation. Show that the function $\bar{\Phi}$ defined on $\lambda^{-1}(\mathcal{D})$ is differentiable with derivative at $X$ of full rank (as a linear map on the tangent space of $\lambda^{-1}(\mathcal{D})$ ). 


\subsection{Transfer of the local approximation}

The goal of this section is to show that locally around $X \in \lambda^{-1}(x)$ the set $\lambda^{-1}(\mathcal{D})$ is an analytic submanifold of $\mathbf{S}^{n}$. We do this in two steps: the first step consists of showing that the $F$-part and the $M$-part of $\mathcal{D}$ give rise to two analytic submanifolds in the spaces $\mathbf{S}_{P_{x}^{F}}^{\left|\mathbb{N}_{n}^{1}\right|}$ and $\mathbf{S}_{P_{x}^{M}}^{\left|\mathbb{N}_{n}^{2}\right|}$ correspondingly. The second step shows that 'intertwining' the two parts preserves this property in the space $\mathbf{S}^{n}$. Suppose the partition $P_{x}=P_{x}^{F} \cup P_{x}^{M}$ has sets

$$
P_{x}^{F}=\left\{I_{1}, \ldots, I_{\kappa}\right\} \quad \text { and } \quad P_{x}^{M}=\left\{I_{\kappa+1}, \ldots, I_{\kappa+m}\right\} .
$$

Lemma 3.11 (Decomposition of $\mathcal{D}$ ). The affine manifold $\mathcal{D}$ can be decomposed as follows

$$
\mathcal{D}=\left\{y^{F} \otimes y^{M}: \quad y^{F} \in \mathcal{D}^{F}, y^{M} \in \mathcal{D}^{M}\right\},
$$

where $\mathcal{D}^{F}$ and $\mathcal{D}^{M}$ are affine manifolds defined by:

$$
\begin{aligned}
\mathcal{D}^{F} & :=\left(\left[T_{\mathcal{M}}^{\mathrm{red}}(x)\right]^{F} \oplus \Delta_{P_{x}^{F}}\right) \cap B\left(x^{F}, \delta_{1}\right), \text { and } \\
\mathcal{D}^{M} & :=\Delta_{P_{x}^{M}} \cap B\left(x^{M}, \delta_{2}\right),
\end{aligned}
$$

where $\left[T_{\mathcal{M}}^{\mathrm{red}}(x)\right]^{F}$ is the $F$-part of the reduced space $T_{\mathcal{M}}^{\mathrm{red}}(x)$. The sets $\mathcal{D}^{F}$ and $\mathcal{D}^{M}$ are locally symmetric and

$$
\operatorname{dim} \mathcal{D}^{F}=d+n^{\text {red }}-m .
$$

Proof. Recalling the definition of $T_{\mathcal{M}}^{\text {red }}(\bar{x})$ and using (2.16) and the right part of (2.13), one sees that for every $y=y^{F} \otimes y^{M} \in T_{\mathcal{M}}^{\text {red }}(x)$ we have $y^{M}=0$. Using the left part of (2.13) with $P=P_{x}$, combined with Proposition 3.8 yields

$$
\begin{aligned}
\mathcal{D} & =\left\{y^{F} \otimes y^{M}: y^{F} \in\left(\left[T_{\mathcal{M}}^{\mathrm{red}}(x)\right]^{F} \oplus \Delta_{P_{x}^{F}}^{\perp}\right) \cap B\left(x^{F}, \delta_{1}\right), y^{M} \in \Delta_{P_{x}^{M}}^{\perp} \cap B\left(x^{M}, \delta_{2}\right)\right\} \\
& =\left\{y^{F} \otimes y^{M}: y^{F} \in\left(\left[T_{\mathcal{M}}^{\mathrm{red}}(x)\right]^{F} \oplus \Delta_{P_{x}^{F}}\right) \cap B\left(x^{F}, \delta_{1}\right), y^{M} \in \Delta_{P_{x}^{M}}^{M} \cap B\left(x^{M}, \delta_{2}\right)\right\},
\end{aligned}
$$

where we used the fact that the ball $B\left(x^{F}, \delta_{1}\right)$ intersects only strata $\Delta_{P^{F}}$ with $P^{F} \succeq P_{x}^{F}$ and similarly for the ball $B\left(x^{M}, \delta_{2}\right)$.

The desired expressions for $\mathcal{D}^{F}$ and $\mathcal{D}^{M}$ follow. By Proposition 3.8, the set $\mathcal{D}$ is invariant under all permutations in $\Sigma_{x}^{n}$. Thus, by Proposition 2.15 (ii), being the $F$-part and the $M$-part of $\mathcal{D}$, the sets $\mathcal{D}^{F}$ and $\mathcal{D}^{M}$ are invariant with respect to the permutations preserving $P_{x}^{F}$ and $P_{x}^{M}$, respectively. We now compute the dimension of $\mathcal{D}^{F}$. Observe that Proposition 3.8 yields

$$
x+T_{\mathcal{M}}(x) \oplus N_{\mathcal{M}}^{\mathrm{red}}(x)=T_{\mathcal{M}}^{\mathrm{red}}(x) \oplus \Delta_{P_{x}}^{\perp \perp}=\left(\left[T_{\mathcal{M}}^{\mathrm{red}}(x)\right]^{F} \oplus \Delta_{P_{x}^{F}}^{\perp \perp}\right) \otimes\left(\{0\} \oplus \Delta_{P_{x}^{M}}^{\perp \perp}\right),
$$

where the zero vector is of dimension $\left|\mathbb{N}_{n}^{2}\right|$. Thus, using (3.9), (3.7), and the fact that $\operatorname{dim} \Delta_{P_{x}^{M}}^{\perp}=m$, we get $d+n^{\text {red }}=\operatorname{dim} \mathcal{D}^{F}+\operatorname{dim} \Delta_{P_{x}^{M}}^{\perp}$, completing the proof.

In the following two lemmas, we show that the two parts of $\mathcal{D}$ lift up to two manifolds $\lambda_{P_{x}^{M}}^{-1}\left(\mathcal{D}^{M}\right)$ and $\lambda_{P_{x}^{F}}^{-1}\left(\mathcal{D}^{F}\right)$. Let us start with the easier case concerning the $M$-part.

Lemma 3.12 (The analytic manifold $\mathcal{S}^{M}$ ). The set $\mathcal{S}^{M}:=\lambda_{P_{x}^{M}}^{-1}\left(\mathcal{D}^{M}\right)$ is an analytic submanifold in $\mathbf{S}_{P_{x}^{M}}^{\left|\mathbb{N}_{n}^{2}\right|}$ with codimension

$$
\sum_{i=1}^{m} \frac{\left|I_{\kappa+i}\right|\left(\left|I_{\kappa+i}\right|+1\right)}{2}-m
$$


Proof. Vectors in $\Delta_{P_{x}^{M}}$ have equal coordinates within each block $I_{\kappa+i}$. Each block lifts to a multiple of the identity matrix (of appropriate dimension). Since the lifting $\lambda_{P_{x}^{M}}^{-1}$ is block-wise, $\mathcal{S}^{M}$ is a direct product of multiples of identity matrices. Hence it is an analytic submanifold with dimension $m$.

Lemma 3.13 (The analytic manifold $\mathcal{S}^{F}$ ). The set $\mathcal{S}^{F}:=\lambda_{P_{x}^{F}}^{-1}\left(\mathcal{D}^{F}\right)$ is an analytic submanifold in $\mathbf{S}_{P_{x}^{F}}^{\left|\mathbb{N}_{n}^{1}\right|}$ with codimension $\left|\mathbb{N}_{n}^{1}\right|-\left(d+n^{\text {red }}-m\right)$.

Proof. By Lemma 3.11, $\mathcal{D}^{F}$ is a locally symmetric, affine submanifold of $\mathbf{R}^{\mathbb{N}_{n}^{1}}$. Our aim is to show that the characteristic partition of $\mathcal{D}^{F}$ is $\mathrm{id}_{\mathbb{N}_{n}^{1}}$. Then applying Theorem 3.7 to $\mathcal{D}^{F}$ shows that $\mathcal{S}^{F}$ is an analytic submanifold of codimension $\left|\mathbb{N}_{n}^{1}\right|-\left(d+n^{\text {red }}-m\right)$.

To this end, fix $\epsilon>0$ and let $\omega \in T_{\mathcal{M}}(\bar{x}) \cap B(0, \epsilon)$ be a vector with the properties stated in Lemma 2.16. That is, vector $w^{F} \in \mathbf{R}^{\mathbb{N}_{n}^{1}}$ is such that every subvector $w_{I_{i}}^{F}$ has distinct coordinates for all $i \in \mathbb{N}_{\kappa}$. By (2.14), there is a unique representation $\omega=\omega_{\perp}+\omega_{\perp \perp}$ for some $\omega_{\perp} \in T_{\mathcal{M}}^{\text {red }}(x)$ and $\omega_{\perp \perp} \in T_{\mathcal{M}}(x) \cap \Delta_{P_{x}}^{\perp}$. Taking the $F$-parts, we have $\omega^{F}=\omega_{\perp}^{F}+\omega_{\perp \perp}^{F}$ with

$$
\omega_{\perp}^{F} \in\left[T_{\mathcal{M}}^{\mathrm{red}}(\bar{x})\right]^{F} \quad \text { and } \quad \omega_{\perp \perp}^{F} \in \Delta_{P_{x}^{F}}^{\perp \perp}
$$

Recall that $P_{x}^{F}=\left\{I_{1}, \ldots, I_{\kappa}\right\}$ and write $\omega_{\perp}^{F}=\omega^{F}-\omega_{\perp \perp}^{F}$. Since subvector $\omega_{I_{i}}^{F}$ has distinct coordinates, while $\left(\omega_{\perp \perp}^{F}\right)_{I_{i}}$ has equal coordinates, we conclude that the subvector $\left(\omega_{\perp}^{F}\right)_{I_{i}}$ has distinct coordinates, for all $i \in \mathbb{N}_{\kappa}$.

Consider now $\mathcal{D}^{F}$. Fix any $x^{F} \in \Delta_{P_{x}^{F}} \cap B\left(x^{F}, \delta_{1}\right)$. Taking $\omega$ close to 0 ensures that $\omega_{\perp}^{F}$ is close to 0 and that all of the coordinates of the vector $\omega_{\perp}^{F}+x^{F}$ are distinct, and moreover $\omega_{\perp}^{F}+x^{F} \in \mathcal{D}^{F}$. All that shows

$$
\mathcal{D}^{F} \cap \Delta_{\mathrm{id}_{\mathbb{N}_{n}^{1}}} \neq \emptyset \text {. }
$$

Thus, the characteristic partition of the affine manifold $\mathcal{D}^{F}$ is $\operatorname{id}_{\mathbb{N}_{n}^{1}}$ as asserted.

We will establish that $\lambda^{-1}(\mathcal{D})$, the intended domain of the local equation of $\lambda^{-1}(\mathcal{M})$, is an analytic manifold, by merging the results of the two preceding lemmas with the help of the following technical result.

Proposition 3.14 (Local canonical split of $\mathbf{S}^{n}$ induced by $P_{x}$ ). Let $x \in \mathcal{M} \cap \mathbf{R}_{\geq}^{n}$. There exist an open neighborhood $W \subset \mathbf{S}^{n}$ of $X \in \lambda^{-1}(x)$ and two analytic maps

$$
\Theta^{F}: W \rightarrow \mathbf{S}_{P_{x}^{F}}^{\mathbb{N}_{n}^{1}} \quad \text { and } \quad \Theta^{M}: W \rightarrow \mathbf{S}_{P_{x}^{M}}^{\mathbb{N}_{n}^{2}},
$$

such that

(i) $\lambda(Y)=\lambda_{P_{x}^{F}}\left(\Theta^{F}(Y)\right) \otimes \lambda_{P_{x}^{M}}\left(\Theta^{M}(Y)\right)$ for all $Y \in W$;

(ii) the Jacobians of the analytic maps $\Theta^{F}$ and $\Theta^{M}$ have full ranks at $X$.

Proof. We apply to each set in the partition $P_{x}=\left\{I_{1}, \ldots, I_{m}\right\}$ a classical result on eigenvalues (see Example 3.98 in [2]) that we recall here. Let $X \in \mathbf{S}^{n}$ have eigenvalues

$$
\lambda_{1}(X) \geq \cdots \geq \lambda_{k-1}(X)>\lambda_{k}(X)=\cdots=\lambda_{k+r-1}(X)>\lambda_{k+r}(X) \geq \cdots \geq \lambda_{n}(X) .
$$

Then, there exist an open neighborhood $W \subset \mathbf{S}^{n}$ of $X$ and an analytic map $\Theta: W \rightarrow \mathbf{S}^{r}$ such that 
(i) for all $Y \in W$, we have $\left\{\lambda_{k}(Y), \ldots, \lambda_{k+r-1}(Y)\right\}=\left\{\lambda_{1}(\Theta(Y)), \ldots, \lambda_{r}(\Theta(Y))\right\}$;

(ii) the Jacobian of $\Theta$ has full rank at $X$.

Recall now that each set $I_{\ell}$ contains consecutive integers and assume without loss of generality that $P_{x}$ is an ordered partition. That is, for all $1 \leq \ell_{1}<\ell_{2} \leq m$ we have that $i \in I_{\ell_{1}}, j \in I_{\ell_{2}}$ implies $i<j$, in other words $\lambda_{i}(X)>\lambda_{j}(X)$. Apply the above result to each $I_{\ell}$ to get open neighborhood $W_{\ell} \subset \mathbf{S}^{n}$ of $X$ and analytic map $\Theta_{\ell}: W_{\ell} \rightarrow \mathbf{S}^{\left|I_{\ell}\right|}$ having a full rank Jacobian. Set $W=\bigcap_{\ell=1}^{m} W_{\ell}$ and put the $F$-pieces and the $M$-pieces together, that is, define the direct products

$$
\Theta^{F}:=\times\left\{\Theta_{\ell}: I_{\ell} \in P_{x}^{F}\right\} \quad \text { and } \quad \Theta^{M}:=\times\left\{\Theta_{\ell}: I_{\ell} \in P_{x}^{M}\right\},
$$

restricting each $\Theta_{\ell}$ to $W$. The order of multiples in the direct products follows the order of the sets $I_{\ell}$ in $P_{x}$. Observe that the above functions satisfy the desired properties.

Theorem 3.15 $\left(\lambda^{-1}(\mathcal{D})\right.$ is a submanifold of $\left.\mathbf{S}^{n}\right)$. The set $\lambda^{-1}(\mathcal{D})$ is an analytic submanifold of $\mathbf{S}^{n}$ around $X \in \lambda^{-1}(x)$, with dimension

$$
\operatorname{dim} \lambda^{-1}(\mathcal{D})=\frac{n(n+1)}{2}+d+n^{\mathrm{red}}-\left|\mathbb{N}_{n}^{1}\right|-\sum_{i=1}^{m} \frac{\left|I_{\kappa+i}\right|\left(\left|I_{\kappa+i}\right|+1\right)}{2}
$$

Proof. By Proposition 3.14, there is a neighborhood $W \subset \mathbf{S}^{n}$ of $X$ and analytic maps $\Theta^{F}$ and $\Theta^{M}$ such that

$$
\lambda(Y)=\lambda_{P_{x}^{F}}\left(\Theta^{F}(Y)\right) \otimes \lambda_{P_{x}^{F}}\left(\Theta^{M}(Y)\right) \quad \text { for all } Y \in W
$$

Set $X^{F}:=\Theta^{F}(X) \in \mathbf{S}_{P_{x}^{F}}^{\left|\mathbb{N}_{n}^{1}\right|}$ and $X^{M}:=\Theta^{M}(X) \in \mathbf{S}_{P_{x}^{M}}^{\left|\mathbb{N}_{n}^{2}\right|}$. Then, (3.16) gives that $x=\lambda(X)=$ $\lambda_{P_{x}^{F}}\left(X^{F}\right) \otimes \lambda_{P_{x}^{M}}\left(X^{M}\right)$, and hence $x^{F}=\lambda_{P_{x}^{F}}\left(X^{F}\right)$ and $x^{M}=\lambda_{P_{x}^{M}}\left(X^{M}\right)$, concluding that $X^{F} \in \mathcal{S}^{F}$ and $X^{M} \in \mathcal{S}^{M}$ (recall Lemma 3.13 and Lemma 3.12). Consider the respective codimensions

$$
\begin{aligned}
& s_{1}:=\operatorname{co-dim} \mathcal{S}^{F}=\left|\mathbb{N}_{n}^{1}\right|-\left(d+n^{\mathrm{red}}-m\right), \text { and } \\
& s_{2}:=\operatorname{co}-\operatorname{dim} \mathcal{S}^{M}=\sum_{i=1}^{m} \frac{\left|I_{\kappa+i}\right|\left(\left|I_{\kappa+i}\right|+1\right)}{2}-m .
\end{aligned}
$$

Since the maps $\Theta^{F}$ and $\Theta^{M}$ have Jacobians of full rank at $X$, they are open around it. By shrinking $W$ if necessary, we may assume there exist analytic maps

$$
\Psi^{F}: \Theta^{F}(W) \rightarrow \mathbf{R}^{s_{1}} \quad \text { and } \quad \Psi^{M}: \Theta^{M}(W) \rightarrow \mathbf{R}^{s_{2}},
$$

with Jacobians having full rank at $X^{F}$ and $X^{M}$ respectively, such that

$$
\Psi^{F}(Y)=0 \Leftrightarrow Y \in \mathcal{S}^{F} \cap \Theta^{F}(W) \quad \text { and } \quad \Psi^{M}(Y)=0 \Leftrightarrow Y \in \mathcal{S}^{M} \cap \Theta^{M}(W) .
$$

We now define a local equation for $\lambda^{-1}(\mathcal{D})$ around $X$ as follows:

$$
\Psi:\left\{\begin{array}{l}
W \longrightarrow \mathbf{R}^{s_{1}} \times \mathbf{R}^{s_{2}} \\
X \longmapsto\left(\Psi^{F} \circ \Theta^{F}\right)(X) \times\left(\Psi^{M} \circ \Theta^{M}\right)(X) .
\end{array}\right.
$$

Indeed, using (3.16), for all $Y \in W$ we have

$$
\Psi(Y)=0 \Longleftrightarrow \lambda(Y)=\lambda_{P_{x}^{F}}\left(\Theta^{F}(Y)\right) \otimes \lambda_{P_{x}^{M}}\left(\Theta^{M}(Y)\right) \in \mathcal{D} \Longleftrightarrow Y \in \lambda^{-1}(\mathcal{D}) .
$$


The fact that the Jacobian of $\Psi$ has full rank at $X$ follows from the chain rule and the fact that all the Jacobians $J \Theta^{F}(X), J \Theta^{M}(X), J \Psi^{F}\left(X^{F}\right)$, and $J \Psi^{M}\left(X^{M}\right)$ are of full rank. Thus, $\Psi$ is an analytic local equation of $\lambda^{-1}(\mathcal{D})$ around $X$, which yields that $\lambda^{-1}(\mathcal{D})$ is a submanifold $\mathbf{S}^{n}$ around $X$. We compute its dimension as follows

$$
\begin{aligned}
\operatorname{dim} \lambda^{-1}(\mathcal{D}) & =\operatorname{dim} \mathbf{S}^{n}-\left(\operatorname{co-} \operatorname{dim} \mathcal{S}^{F}+\operatorname{co-} \operatorname{dim} \mathcal{S}^{M}\right) \\
& =\frac{n(n+1)}{2}+d+n^{\mathrm{red}}-\left|\mathbb{N}_{n}^{1}\right|-\sum_{i=1}^{m} \frac{\left|I_{\kappa+i}\right|\left(\left|I_{\kappa+i}\right|+1\right)}{2},
\end{aligned}
$$

where (3.17) and (3.18) were used.

Theorem 3.15 is an important intermediate result for the forthcoming Section 3.4, which contains the final step of the proof. Nonetheless, in the following particular case, Theorem 3.15 allows us to conclude directly.

Example 3.16 (Lift of strata). Fix a partition $P^{\circ} \in \Pi_{n}$. Then, the set

$$
\mathcal{M}:=\Delta_{P^{\circ}} \cup\left(\bigcup_{P \propto P^{\circ}} \Delta_{P}\right)
$$

is a locally symmetric manifold with characteristic permutation $P^{\circ}$, see [7]. Suppose in addition that the sets in $P^{\circ}$ contain consecutive integers, then $\mathcal{M} \cap \mathbf{R}_{\geq}^{n} \neq \emptyset$. For any $x \in \mathcal{M} \cap \mathbf{R}_{\geq}^{n}$ we have $N_{\mathcal{M}}^{\text {red }}(x)=\{0\}$, that is $n^{\text {red }}=0$. This means that the affine manifolds $\mathcal{M}$ and $\mathcal{D}$ coincide locally around $x$, see (3.8). In this case Theorem 3.15 shows directly that $\lambda^{-1}(\mathcal{M})$ is a manifold in $\mathbf{S}^{n}$ with dimension given by (3.15). At first glance, it appears that the dimension depends on the particular choice of $x$. But since $P_{x}=P_{\circ}$ or $P_{x} \prec P_{\circ}$, by Proposition 2.15 (ii) we have $P_{x}^{M}=P_{\circ}^{M}=$ : $\left\{I_{\kappa+1}^{\circ}, \ldots, I_{\kappa+m}^{\circ}\right\}$. Let $P_{\circ}^{F}=\left\{I_{1}^{\circ}, \ldots, I_{\kappa}^{\circ}\right\}$. Using that $n=\left|\mathbb{N}_{n}^{1}\right|+\left|\mathbb{N}_{n}^{2}\right|=\sum_{i=1}^{\kappa}\left|I_{i}^{\circ}\right|+\sum_{i=\kappa+1}^{\kappa+m}\left|I_{i}^{\circ}\right|$, one can verify that $(3.15)$ becomes

$$
\operatorname{dim} \lambda^{-1}(\mathcal{M})=d+\sum_{1 \leq i<j \leq \kappa+m}\left|I_{i}^{\circ} \| I_{j}^{\circ}\right|
$$

Thus, according to $(3.1)$, we have $\operatorname{dim} \lambda^{-1}(\mathcal{M})=\operatorname{dim} \lambda^{-1}\left(\Delta_{P_{\mathrm{o}}}\right)$. This is a particular case of the forthcoming general formula (3.21).

In the situation of Example 3.16 the manifold $\mathcal{M}$ has a trivial reduced normal space. The following remark sheds more light on this aspect.

Remark 3.17 (Trivial reduced normal space). Let $\mathcal{M}$ be a locally symmetric manifold, with characteristic partition $P_{*}$ and let $x \in \mathcal{M} \cap \mathbf{R}_{\geq}^{n}$. Then, by (2.21) and (2.23), it can be seen that

$$
N_{M}^{\mathrm{red}}(x)=\{0\} \Longleftrightarrow \mathcal{M} \cap B(x, \delta)=\left(x+T_{\mathcal{M}}(x)\right) \cap B(x, \delta) \text { for some } \delta>0 .
$$

Inclusion (2.9) shows that $x \in \Delta_{P_{*}}^{\perp}$ and together with (2.10) we get that $\left(x+T_{\mathcal{M}}(x)\right) \subset \Delta_{P_{*}}^{\perp}$. Thus, we obtain

$$
N_{M}^{\mathrm{red}}(x)=\{0\} \Longleftrightarrow \mathcal{M} \cap B(x, \delta)=\Delta_{P_{*}}^{\perp} \cap B(x, \delta) \text { for some } \delta>0 .
$$




\subsection{Transfer of local equations, proof of the main result}

This section contains the last step of our argument. We show that (3.12) is indeed a local equation of $\mathcal{M}$ around $X \in \lambda^{-1}(x)$.

Lemma 3.18 (The Jacobian of $\bar{\Phi}$ ). The map $\bar{\Phi}$ defined in (3.12) is of class $C^{k}$ at $X \in \lambda^{-1}(x)$. Denoting the differential of $\bar{\Phi}$ at $X$ by

$$
D \bar{\Phi}(X): T_{\lambda^{-1}(\mathcal{D})}(X) \longrightarrow N_{\mathcal{M}}^{\mathrm{red}}(x),
$$

we have for any direction $H \in T_{\lambda^{-1}(\mathcal{D})}(X)$ :

$$
D \bar{\Phi}(X)[H]=D \phi\left(\bar{\pi}_{T}(\lambda(X))\right)\left[\pi_{T}\left(\operatorname{diag}\left(U H U^{\top}\right)\right)\right]-\pi_{N}^{\mathrm{red}}\left(\operatorname{diag}\left(U H U^{\top}\right)\right),
$$

where $U \in \mathbf{O}^{n}$ is such that $X=U^{\top}(\operatorname{Diag} \lambda(X)) U$.

Proof. We deduce from Theorem 2.17 that for any $\sigma \in \Sigma_{x}^{n}$ and $y \in \mathcal{D}$ we have

$$
\left(\phi \circ \bar{\pi}_{T}\right)(\sigma y)=\left(\phi \circ \bar{\pi}_{T}\right)(y) .
$$

In addition, the gradient of the $i$-th coordinate function $\phi_{i} \circ \bar{\pi}_{T}$ at $x$ applied to any direction $h \in T_{\mathcal{D}}(x)=T_{\mathcal{M}}^{\mathrm{red}}(x) \oplus \Delta_{P_{x}^{\perp}}^{\perp}$, see Proposition 3.8, yields

$$
\nabla\left(\phi_{i} \circ \bar{\pi}_{T}\right)(x)[h]=\nabla \phi_{i}\left(\bar{\pi}_{T}(x)\right)\left[\pi_{T}(h)\right] .
$$

Thus, by Theorem 2.2, we obtain the following expression for the gradient at $X$ of the function $\phi_{i} \circ \bar{\pi}_{T} \circ \lambda$ applied to the direction $H \in T_{\lambda^{-1}(\mathcal{D})}(X)$

$$
\nabla\left(\phi_{i} \circ \bar{\pi}_{T} \circ \lambda\right)(X)[H]=\nabla \phi_{i}\left(\bar{\pi}_{T}(\lambda(X))\right)\left[\pi_{T}\left(\operatorname{diag}\left(U H U^{\top}\right)\right)\right] \quad \text { for } i \in \mathbb{N}_{n},
$$

where $U \in \mathbf{O}^{n}$ is such that $X=U^{\top}(\operatorname{Diag} \lambda(X)) U$. By Lemma 3.9 , the projection $\bar{\pi}_{N}^{\text {red }}$ is locally symmetric at $x$. Thus, by (2.4) we have

$$
J\left(\bar{\pi}_{N}^{\mathrm{red}} \circ \lambda\right)(X)[H]=J \bar{\pi}_{N}^{\mathrm{red}}(\lambda(X))\left[\operatorname{diag}\left(U H U^{\top}\right)\right]=\pi_{N}^{\mathrm{red}}\left(\operatorname{diag}\left(U H U^{\top}\right)\right) .
$$

Formula (3.19) is obtained.

Next, we show that the differential of $\bar{\Phi}$ at $X$ is of full rank. We accomplish this without actually computing the tangent space of the manifold $\lambda^{-1}(\mathcal{D})$ at $X$. Instead we show that the tangent space is sufficiently rich to guarantee surjectivity.

Lemma 3.19 (Surjectivity of $D \bar{\Phi}(X)$ ). The differential of $\bar{\Phi}$ at $X$

$$
D \bar{\Phi}(X): T_{\lambda^{-1}(\mathcal{D})}(X) \longrightarrow N_{\mathcal{M}}^{\mathrm{red}}(x)
$$

is onto. Hence, it has a full rank.

Proof. Let $U \in \mathbf{O}^{n}$ be such that $X=U^{\top}(\operatorname{Diag} \lambda(X)) U$. The tangent space of $\mathbf{O}^{n}$ at $U$ is

$$
\{U A: A \text { is an } n \times n \text { skew-symmetric matrix }\} .
$$

Thus, for any $n \times n$ skew symmetric matrix $A$ there exists an analytic curve $t \mapsto U(t) \in \mathbf{O}^{n}$ such that

$$
U(0)=U \quad \text { and } \quad \dot{U}(0):=\frac{d}{d t} U(0)=U A .
$$


Fix now any vector $h \in N_{\mathcal{M}}^{\text {red }}(x)$. Consider the curve $t \mapsto U(t)^{\top}(\operatorname{Diag}(x+t h)) U(t)$. For all values of $t$ close to zero, this curve lies in $\lambda^{-1}(\mathcal{D})$ because $x+t h$ lies in $\mathcal{D}$. Introduce the vector $x_{t}$ made of the entries of $x+t h$ reordered in decreasing way. Since the space $N_{\mathcal{M}}^{\text {red }}(x)$ is invariant under all permutations $\sigma \in \Sigma_{x}^{n}$ we see that $x_{t}$ lies in $x+N_{\mathcal{M}}^{\mathrm{red}}(x)$, for $t$ close to zero. The derivative of this curve at $t=0$ (i.e. a tangent vector in $\left.T_{\lambda^{-1}(\mathcal{D})}(X)\right)$ is

$$
\begin{aligned}
H & :=\dot{U}(0)^{\top}(\operatorname{Diag} x) U(0)+U(0)^{\top}(\operatorname{Diag} h) U(0)+U(0)^{\top}(\operatorname{Diag} x) \dot{U}(0) \\
& =-A U^{\top}(\operatorname{Diag} x) U+U^{\top}(\operatorname{Diag} h) U+U^{\top}(\operatorname{Diag} x) U A,
\end{aligned}
$$

using that $A^{\top}=-A$. Substituting the above expression of $H$ into (3.19), and using the fact that $U U^{\top}=U^{\top} U=I$ and that $U A U^{\top}(\operatorname{Diag} x)$ and $(\operatorname{Diag} x) U A U^{\top}$ have the same diagonal we obtain

$$
D \bar{\Phi}(X)[H]=-h .
$$

This shows that $D \bar{\Phi}(X)$ is surjective onto $N_{\mathcal{M}}^{\text {red }}(x)$, completing the proof.

Theorem 3.20 (The main result). Let $\mathcal{M}$ be a locally symmetric $C^{k}$ submanifold of $\mathbf{R}^{n}$ of dimension d. Then $\lambda^{-1}(\mathcal{M})$ is a $C^{k}$ submanifold of $\mathbf{S}^{n}$ of dimension

$$
\operatorname{dim} \lambda^{-1}(\mathcal{M})=d+\sum_{1 \leq i<j \leq m^{*}}\left|I_{i}^{*}\right|\left|I_{j}^{*}\right|
$$

where $P_{*}=\left\{I_{1}^{*}, \ldots, I_{m^{*}}^{*}\right\}$ is the characteristic partition of $\mathcal{M}$. Here $k \in\{2,3, \ldots, \infty, \omega\}$.

Proof. Fix any $x \in \mathcal{M} \cap \mathbf{R}_{>}^{n}$ and $X \in \lambda^{-1}(x)$ and consider the spectral function $\bar{\Phi}$ introduced in (3.12). Equation (3.13) shows that $\bar{\Phi}$ is a local equation of $\mathcal{M}$. Lemmas 3.18 and 3.19 prove that $\bar{\Phi}$ is a $C^{k}$ local equation of $\lambda^{-1}(\mathcal{M})$ around $X$. Thus, $\lambda^{-1}(\mathcal{M})$ is a $C^{k}$ submanifold of $\mathbf{S}^{n}$ around $X$. Moreover, the dimension of $\lambda^{-1}(\mathcal{M})$ is

$$
\operatorname{dim} \lambda^{-1}(\mathcal{M})=\operatorname{dim} \lambda^{-1}(\mathcal{D})-\operatorname{dim}\left(N_{\mathcal{M}}^{\mathrm{red}}(x)\right) .
$$

Suppose that (3.14) holds. By Proposition 2.15 (ii), we have that $P_{x}^{M}=P_{*}^{M}$. So suppose that $I_{\kappa+i}=I_{m^{*}-m+i}^{*}$ for all $i=1, \ldots, m$. Recall that $n=\left|\mathbb{N}_{n}^{1}\right|+\left|\mathbb{N}_{n}^{2}\right|$ and that $\sum_{i=1}^{m}\left|I_{\kappa+i}\right|=n-\left|\mathbb{N}_{n}^{1}\right|$. Using (3.6) and Theorem 3.15, we get

$$
\begin{aligned}
\operatorname{dim} \lambda^{-1}(\mathcal{M}) & =d+\frac{n(n+1)}{2}-\left|\mathbb{N}_{n}^{1}\right|-\sum_{i=1}^{m} \frac{\left|I_{\kappa+i}\right|\left(\left|I_{\kappa+i}\right|+1\right)}{2} \\
& =d+\frac{n^{2}}{2}-\frac{\left|\mathbb{N}_{n}^{1}\right|}{2}-\sum_{i=1}^{m} \frac{\left|I_{\kappa+i}\right|^{2}}{2} \\
& =d+\frac{n^{2}}{2}-\frac{\left|\mathbb{N}_{n}^{1}\right|}{2}-\frac{1}{2}\left(\sum_{i=1}^{m}\left|I_{m^{*}-m+i}^{*}\right|\right)^{2}+\sum_{1 \leq i<j \leq m}\left|I_{m^{*}-m+i}^{*}\right|\left|I_{m^{*}-m+j}^{*}\right| \\
& =d+\frac{\left|\mathbb{N}_{n}^{1}\right|\left(\left|\mathbb{N}_{n}^{1}\right|-1\right)}{2}+\left|\mathbb{N}_{n}^{1}\right|\left(n-\left|\mathbb{N}_{n}^{1}\right|\right)+\sum_{1 \leq i<j \leq m}\left|I_{m^{*}-m+i}^{*}\right|\left|I_{m^{*}-m+j}^{*}\right| \\
& =d+\sum_{1 \leq i<j \leq m^{*}}\left|I_{i}^{*}\right|\left|I_{j}^{*}\right|,
\end{aligned}
$$

the last equality uses the fact that all the sets in $P_{*}^{F}=\left\{I_{1}^{*}, \ldots, I_{m^{*}-m}^{*}\right\}$ have size one. 
Notice that the dimension $(3.21)$ of $\lambda^{-1}(\mathcal{M})$ depends only on the dimension of the underlying manifold $\mathcal{M}$ and its characteristic permutation $P_{*}$. This is not the case with the dimension (3.15) of $\lambda^{-1}(\mathcal{D})$ which also depends on the active partition $P_{x}$ (by $n^{\text {red }}, \kappa$ and $m$ ).

Remark 3.21 (Comment for the case of $C^{1}$-manifolds). The case of a locally symmetric $C^{1}$ manifold $\mathcal{M}$ is compromised by [7, Lemma 3.13] (Determination of isometries), which uses the intrinsic Riemannian structure of $\mathcal{M}$, requiring thus, a $C^{2}$ regularity. Lemma 3.13 from [7] had been subsequently used to obtain the reduction of the ambient space for the tangential parametrization of $\mathcal{M}$, which has been one of the main ingredients in establishing Theorem 2.17.

Let us now give a few applications of the main result. It is known that the set of all symmetric matrices in $\mathbf{S}^{n}$ of rank $k$ is an analytic manifold, see for example [9, Proposition 1.14, p.133]. This also follows from our main result, together with the formula for its dimension.

Example 3.22 (Matrices of constant rank). Suppose $\mathcal{M}=\left\{x \in \mathbf{R}^{n}: x\right.$ has exactly $n-k$ zeros $\}$, then $\lambda^{-1}(\mathcal{M})=\left\{A \in S^{n}: \operatorname{rank} A=k\right\}$. Fix a subset $J$ of $\{1,2, \ldots, n\}$ with $n-k$ consecutive elements and define $\mathcal{M}^{\prime}:=\left\{x \in \mathbf{R}^{n}: x_{i}=0, i \in J\right\}$, a connected component of $\mathcal{M}$. Then, $\operatorname{dim} \mathcal{M}^{\prime}=k$ and the characteristic partition of $\mathcal{M}^{\prime}$ is $P_{*}=\{i: i \in J\} \cup\{\{i\}: i \notin J\}$. By Theorem 3.20, $\lambda^{-1}(\mathcal{M})$ is an analytic submanifold of $\mathbf{S}^{n}$ with dimension

$$
\operatorname{dim} \lambda^{-1}(\mathcal{M})=\operatorname{dim} \lambda^{-1}\left(\mathcal{M}^{\prime}\right)=k+\frac{k(k-1)}{2}+k(n-k)=\frac{k(2 n-k+1)}{2} .
$$

In particular, the dimension of rank-one matrices $(k=1)$ is $n$, while the dimension of the invertible matrices $(k=n)$ is $\left(\begin{array}{l}n \\ 2\end{array}\right)$.

Example 3.23 (The unit shpere). Consider the unit sphere in $\mathbf{R}^{n}$ :

$$
\mathcal{M}:=\left\{x \in \mathbf{R}^{n}: x_{1}^{2}+\cdots+x_{n}^{2}=1\right\} .
$$

It is a symmetric analytic manifold of dimension $n-1$ and characteristic partition $P_{*}=\{\{i\}: i=$ $1, \ldots, n\}$. By Theorem 3.20, $\lambda^{-1}(\mathcal{M})$ is an analytic submanifold of $\mathbf{S}^{n}$ with dimension

$$
\operatorname{dim} \lambda^{-1}(\mathcal{M})=(n-1)+\left(\begin{array}{l}
n \\
2
\end{array}\right)=\left(\begin{array}{c}
n+1 \\
2
\end{array}\right)-1
$$

Indeed, it is easy to see that

$$
\lambda^{-1}(\mathcal{M})=\left\{A \in \mathbf{S}^{n}:\|\lambda(A)\|=1\right\}=\left\{A \in \mathbf{S}^{n}:\|A\|=1\right\} .
$$

That is, $\lambda^{-1}(\mathcal{M})$ is the unit sphere in $\mathbf{S}^{n}$.

Remark 3.24 (The case $\left|\mathbb{N}_{n}^{1}\right| \in\{0,1\}$ ). If $\mathcal{M}$ is a connected, submanifold of $\mathbf{R}^{n}$ of dimension $d$, such that $\left|\mathbb{N}_{n}^{1}\right| \in\{0,1\}$, then $\mathcal{M} \subset \Delta_{P_{*}}$. The same arguments as in Example 3.1 allow to conclude that $\lambda^{-1}(\mathcal{M})$ is a spectral manifold of dimension given by $(3.1)$.

\section{Acknowledgment}

Our gratitude goes to an anonymous referee for his or her time and valuable comments. The authors wish to thank Vestislav Apostolov (UQAM, Montreal, Canada), Vincent Beck (ENS Cachan, France), Matthieu Gendulphe (University of Fribourg, Switzerland), and Joaquim Roé (UAB, Barcelona, Spain) for interesting and useful discussions on early stages of this work. We especially thank Adrian Lewis (Cornell University, Ithaca, USA) for useful discussions, and in particular for pointing out Proposition 1.1. 


\section{References}

[1] Ball, M. J., Differentiability properties of symmetric and isotropic functions, Duke Math. J. 51 (1984), 699-728.

[2] Bonnans, F. And Shapiro, A., Perturbation Analysis of Optimization Problems, (Springer, 2000).

[3] Dadok, J., On the $C^{\infty}$ Chevalley's theorem, Advances in Mathematics 44 (1982), 121-131.

[4] Danillidis, A., Drusvyatskiy, D. And Lewis, A. S., Orthogonal Invariance and Identifiability, 21 p. (Submitted, April 2013)

[5] Danillidis, A., Lewis, A.S., Malick, J. And Sendov, H., Prox-regularity of spectral functions and spectral sets, J. Convex Anal. 15 (2008), 547-560.

[6] Danillidis, A., Malick, J. And Sendov, H., Locally symmetric submanifolds lift to spectral manifolds, Preprint UAB 23 (2009), 43 p., and Preprint Arxiv 1212.3936 (available at http://arxiv.org/abs/1212.3936)

[7] Danilidis, A., Malick, J. And Sendov, H., On the structure of locally symmetric manifolds, 24 p. (Submitted, April 2013).

[8] Do Carmo, M.P., Riemannian Geometry, (Birkhäuser, 1993).

[9] Helmke, U. And Moore, J.B., Optimization and dynamical systems, Second edition, (New York, Springer, 1996).

[10] Hiriart-Urruty, J.-B. And Ye, D., Sensitivity analysis of all eigenvalues of a symmetric matrix, Numer. Math. 70 (1992), 45-72.

[11] Kato, T., A Short Introduction to Perturbation Theory for Linear Operators, (SpringerVerlag, Berlin, 1976).

[12] Kobayashi, S. and Nomizu, K., Foundations of Differential Geometry. Vol I, (John Wiley \& Sons, New York-London, 1963).

[13] Lewis, A.S., Derivatives of spectral functions, Math. Oper. Res. 21 (1996), 576-588.

[14] Lewis, A.S., Nonsmooth analysis of eigenvalues, Math. Programming 84 (1999), 1-24.

[15] Lewis, A.S. And Sendov, H., Twice differentiable spectral functions, SIAM J. Matrix Anal. Appl. 23 (2001), 368-386.

[16] Orsi, R., Helmke, U. And Moore, J.B., A Newton-like method for solving rank constrained linear matrix inequalities, Automatica 42 (2006), 1875-1882.

[17] Poliquin, R.A. and Rockafellar, R.T., Prox-regular functions in variational analysis. Trans. Amer. Math. Soc. 348 (1996) 1805-1838.

[18] Sendov, H., The higher-order derivatives of spectral functions, Linear Algebra Appl. 424 (2007), 240-281.

[19] ŠılhavÝ, M., Differentiability properties of isotropic functions, Duke Math. J. 104 (2000), 367-373. 
[20] Sylverster, J., On the differentiability of $O(n)$ invariant functions of symmetric matrices, Duke Math. J. 52(2) (1985), 475-483.

[21] Tropp, J.A., Dhillon, I.S., Heath, R.W. and Strohmer, T., Designing structured tight frames via in alternating projection method, IEEE Trans. on Inf. Theory 51 (2005), 188-209.

[22] Tsing, N.-K., FAn, M.K.H. And Verriest, E.I., On analyticity of functions involving eigenvalues, Linear Algebra Appl. 207 (1994), 159-180.

\section{Aris DANIILIDIS}

Departament de Matemàtiques, C1/364

Universitat Autònoma de Barcelona

E-08193 Bellaterra (Cerdanyola del Vallès), Spain (on leave) ;

and

DIM-CMM, Universidad de Chile

Blanco Encalada 2120, piso 5, Santiago, Chile;

E-mail: arisd@mat.uab.es ; arisd@dim.uchile.cl

http://www.dim.uchile.cl/ arisd

Research supported by the grant MTM2011-29064-C01 (Spain)

and by the FONDECYT Regular grant No 1130176 (Chile).

\section{Jérôme MALICK}

CNRS, Laboratoire J. Kunztmann

Grenoble, France

E-mail: jerome.malick@inria.fr

http://bipop.inrialpes.fr/people/malick/

\section{Hristo SENDOV}

Department of Statistical \& Actuarial Sciences

The University of Western Ontario, London, Ontario, Canada

E-mail: hssendov@stats.uwo.ca

http://www.stats . uwo.ca/faculty/hssendov/Main.html

Research supported by the NSERC of Canada. 